\title{
IRREDUCIBLE REPRESENTATIONS OF THE GROUP OF UNIPOTENT MATRICES OF ORDER 4 OVER INTEGERS
}

\author{
IULIYA BELOSHAPKA
}

\begin{abstract}
We study a coarse moduli space of irreducible representations of the group of unipotent matrices of order 4 over the ring of integers which have finite weight. All such representations are known to be monomial (see [2]). To describe a coarse moduli space of such representations, we need to study pairs of subgroups and their characters, which induce nonisomorphic irreducible representations. We obtain a full classification of such pairs and, respectively, a coarse moduli space.
\end{abstract}

\section{INTRODUCTION}

Moduli spaces of irreducible representations of finitely generated nilpotent groups are supposed to be used in questions related to $L$-functions of varieties over finite fields (see [10]). It does not seem reasonable to study such moduli spaces in full generality, so one should restrict the class of all irreducible objects. Brown in [6] introduced the notion of a finite weight representation. A representation $\pi$ of a group $G$ has finite weight if there is a subgroup $H \subset G$ and a character $\chi$ of $H$ such that the vector space $\operatorname{Hom}_{H}\left(\chi,\left.\pi\right|_{H}\right)$ is non-zero and finite-dimensional. A representation $\pi$ is called monomial, if there exist a subgroup $H \subset G$ and a character $\chi: H \rightarrow \mathbb{C}^{*}$ such that $\pi \simeq \operatorname{ind}_{H}^{G}(\chi)$. In the plenary lecture at ICM 2010, Parshin conjectured that irreducible representations of finitely generated nilpotent groups are monomial if and only if they have finite weight (see [10, $\S 5.4(\mathrm{i})]$ for details). The conjecture was proven in a joint work with Gorchinskiy [2]. This allows us to approach the moduli problem of irreducible representations $\pi$ which have finite weight, since they always correspond to certain pairs $(H, \chi)$ such that $\pi \simeq \operatorname{ind}_{H}^{G}(\chi)$.

Parshin and Arnal have studied in detail the case of the Heisenberg group over the integers [1. For this group, they constructed a parameter space (i.e., a coarse moduli space) of complex irreducible representations which have finite weight. It turns out that the parameter space consists of two parts, corresponding to finite-dimensional irreducible representations and infinitedimensional ones. The first one is a countable disjoint union of copies of $\mathbb{C}^{*} \times \mathbb{C}^{*}$. The second one, in turn, is a countable disjoint union of elliptic fibrations over $\mathbb{C}^{*} \backslash S^{1}$ and components which do not have a complex variety 
structure (see [10], 9] for details). The question was also studied in the case of mixed real and integer coefficients, which was motivated by the theory of two-dimensional local fields 3 .

The construction looks similar to Kirillov's orbit method [8] for connected real or complex nilpotent Lie groups. Attempts to extend Kirillov's method to $p$-adic nilpotent groups were made in [7]. Also, there exists an analogue of Kirillov's character formula for the discrete Heisenberg group.

Our main result is the construction of a coarse moduli space of irreducible representations of the group of unipotent $4 \times 4$ matrices over integers which have finite weight (see Theorem 10.1 and Table 11). We denote this group by $G$. In other words, we provide a full classification of pairs $(H, \chi)$ such that corresponding representations $\operatorname{ind}_{H}^{G}(\chi)$ are irreducible.

THEOREM. There is a one-to-one correspondence between the following spaces:

1. The union of the total spaces of the following bundles: $X_{1,1} \rightarrow \Xi_{1,1}$, $X_{2,0} \rightarrow \Xi_{2,0}, X_{2,1} \rightarrow \Xi_{2,1}, X_{1,2} \rightarrow \Xi_{1,2}, X_{2,2} \rightarrow \Xi_{2,2}$, and $X_{3,2} \rightarrow \Xi_{3,2}$.

2. A coarse moduli space of irreducible representations for the group of unipotent matrices of order 4 with integer entries which have finite weight.

A map from $X_{r_{1}, r_{2}} \rightarrow \Xi_{r_{1}, r_{2}}$ to the set of irreducible monomial representations is defined as follows:

$$
(H, \chi) \longmapsto \operatorname{ind}_{H}^{G}(\chi) .
$$

First steps towards the moduli space problem were made in [4]. The coarse moduli space has a natural iterated structure of a bundle over the set of certain subgroups $H \subset G$ (see Theorem 10.1). It turns out that the number of isomorphism classes of irreducible finite weight representations of discrete nilpotent groups increases very rapidly, while a nilpotency class increments only by one. Namely, for the Heisenberg group over the integers, there are only two substantially different cases of weight pairs $(H, \chi)$ which correspond to irreducible monomial representations. In turn, there are over 50 different cases for the group $G$, which makes our classification quite technical and lengthy. The developed techniques may be used for further generalizations to finitely generated nilpotent groups of higher nilpotency classes.

The paper is organized as follows. In Section 2, we provide results which concern an arbitrary finitely generated nilpotent group, some of which are well known. In Subsection 2.1 we collect well-known formulas for endomorphisms of finitely induced representations, based on Frobenius reciprocity and 
Mackey's formula. Also, we introduce a notion of an irreducible weight pair. In Subsection 2.2 we recall a result from [2] which relates ireducibility and Schur irreducibility of finitely induced representations. In Subsection 2.3 we prove a result which concerns irreducible weight pairs with abelian subgroups of finitely generated torsion-free nilpotent groups. In Subsection 2.4 we recall a criterion from [2] for irreducible weight pairs to be equivalent. We also prove there a result which concerns equivalent weight pairs with subgroups which

have the same radical subgroup. In Subsection 2.5 we recall a definition of ranks of finitely generated nilpotent groups.

All the other sections concern the group of unipotent matrices of order 4 over the ring of integers.

In Section 3 we list the ranks of subgroups which may appear for this group and which provide irreducible monomial representations. The next six sections are organised in a similar way as we are dealing with six possible cases of ranks of subgroups. In each of those sections first we describe generators of subgroups of given ranks, and then we obtain the conditions on a character of a subgroup that the weight pair is irreducible. After that, we study equivalent irreducible weight pairs such that a subgroup is the same, but characters are different. Then we find all irreducible weight pairs which are equivalent to a given one such that subgroups in those pairs are different.

Section 10 sums up all the possible cases of ranks of subgroups in a classification theorem, which is the main result of the paper.

\section{Preliminaries}

We recall some well-known facts. Let $G$ be an arbitrary group, and $H$ be a subgroup of a group $G$. We use notation from [2].

\subsection{Endomorphisms of finitely induced representations.}

Definition 2.1. Let $S(H) \subset G$ be the set of all elements $g \in G$ such that the index of $H^{g} \cap H$ in $H$ is finite.

Let $\chi: H \rightarrow \mathbb{C}^{*}$ be a character of a subgroup $H$.

Proposition 2.2. There is a canonical isomorphism of vector spaces

$$
\operatorname{End}_{G}\left(\operatorname{ind}_{H}^{G}(\chi)\right) \simeq \bigoplus_{\bar{g} \in H \backslash S(H) / H} \operatorname{Hom}_{H^{g} \cap H}\left(\left.\chi\right|_{H^{g} \cap H},\left.\chi^{g}\right|_{H^{g} \cap H}\right) .
$$

Proposition 2.2 motivates the following definition.

Definition 2.3. Let $S(H, \chi) \subset G$ be the set of all elements $g \in S(H)$ such that

$$
\operatorname{Hom}_{H^{g} \cap H}\left(\left.\chi\right|_{H^{g} \cap H},\left.\chi^{g}\right|_{H^{g} \cap H}\right) \neq 0,
$$




$$
\begin{gathered}
\text { or, equivalently, } \\
\left.\chi\right|_{H^{g} \cap H}=\left.\chi^{g}\right|_{H^{g} \cap H} .
\end{gathered}
$$

As an immediate corollary from the Proposition 2.2, we obtain a canonical isomorphism of vector spaces

$$
\operatorname{End}_{G}\left(\operatorname{ind}_{H}^{G}(\chi)\right) \simeq \bigoplus_{H \backslash S(H, \chi) / H} \mathbb{C} .
$$

Definition 2.4. An irreducible weight pair is a pair $(H, \chi)$ such that $H \subset G$ is a subgroup, $\chi$ is a character of $H$, and $\operatorname{ind}_{H}^{G}(\chi)$ is an irreducible representation.

Remark 2.5. Unfortunately, this definition is in a way an abuse of notation. In the paper [2] an irreducible pair was defined as a pair $(H, \rho)$, where $H \subset G$ is a subgroup and $\rho$ is a (non-zero) finite-dimensional irreducible representation of $H$.

2.2. Irreducibility vs. Schur irreducibility. We recall that a representation $\pi$ is called Schur-irreducible if $\operatorname{End}_{G}(\pi)=\mathbb{C}$.

We also recall that a generalization of Schur's lemma to countable groups holds true. Namely, any countably dimensional irreducible representation over $\mathbb{C}$ of an arbitrary group is Schur-irreducible (see, e.g., [5, Claim 2.11]).

Remark 2.6. For an irreducible weight pair $(H, \chi)$, the centralizer $C_{G}(H)=$ $\{g \in G \mid[g, h]=1$ for any $h \in H\}$ is a subgroup of $H$, and, in particular, the center $Z(G)$ is contained in $H$.

Proof. Clearly, $C_{G}(H) \subset S(H, \chi)$. For an irreducible weight pair $(H, \chi)$, it follows from Schur's lemma that $\operatorname{End}_{G}(\pi)=\mathbb{C}$, and then $S(H, \chi)=H$.

Now let $G$ be a finitely generated nilpotent group.

We will essentially use the following theorem. It allows us to replace irreducibility of representations with Schur-irreducibility, which is much easier to check.

Theorem 2.7. [2, Theorem 3.14] Let $H$ be a subgroup of a finitely generated nilpotent group $G$. Let $\rho$ be an irreducible complex representation of $H$ such that the finitely induced representation $\operatorname{ind}_{H}^{G}(\rho)$ satisfies $\operatorname{End}_{G}\left(\operatorname{ind}_{H}^{G}(\rho)\right)=\mathbb{C}$. Then the representation $\operatorname{ind}_{H}^{G}(\rho)$ is irreducible.

We will use this theorem for $\rho=\chi$, a one-dimensional representation of $H$. Remark 2.8. Under the conditions of Theorem 2.7, a representation $\operatorname{ind}_{H}^{G}(\rho)$ is irreducible if and only if $S(H, \chi)=H$. 
2.3. Irreducible weight pairs with abelian subgroups. Let us denote by $\gamma_{1}(G)=[G, G], \gamma_{k}(G)=\left[G, \gamma_{k-1}(G)\right]$.

Definition 2.9. Let $H^{*}$ be the smallest subgroup of $G$ with the following properties: $H^{*}$ contains $H$ and if an element $g \in G$ satisfies $g^{i} \in H^{*}$ for some positive integer $i$, then $g \in H^{*}$.

A subgroup $H$ is called isolated if $H=H^{*}$.

For elements $g, h \in G$ we will denote a conjugated element $h g h^{-1}$ by $g^{h}$.

Proposition 2.10. Let $G$ be a finitely generated torsion-free nilpotent group, such that torsion of $\left(G / \gamma_{k}(G)\right)$ is trivial for every $k$. Let $H \subset G$ be an abelian subgroup of $G$. If $H$ form an irreducible weight pair $(H, \chi)$ for some character $\chi: H \rightarrow \mathbb{C}^{*}$, then $H$ is isolated.

To prove the proposition, we need the following simple lemma:

Lemma 2.11. Let $g, h$ be elements of $G$ such that $k$ is the maximal number that $[h, g] \in \gamma_{k}(G)$. Then there is a homomorphism $\psi:\langle g\rangle \rightarrow G / \gamma_{k+1}(G)$, which maps $g^{i}$ to $\left[h, g^{i}\right]$.

Proof. Follows directly from the formula

$$
\left[h, g^{i} g^{j}\right]=\left[h, g^{i}\right]\left[h, g^{j}\right]^{g^{i}}=\left[h, g^{i}\right]\left[h, g^{j}\right]\left[g^{i},\left[h, g^{j}\right]\right]
$$

since $\left[g^{i},\left[h, g^{j}\right]\right]$ is in $\gamma_{k+1}(G)$.

Now we can prove the Proposition 2.10.

Proof. Assume the opposite, then there exists an element $g \in H^{*} \backslash H$. That is $g^{n} \in H$ for some integer $n$. Since $C_{G}(H)=H$, there exists an element $h \in H$ such that $g$ does not commute with $h$. Since $G$ is nilpotent, there exists $k$ such that $[h, g] \in \gamma_{k}(G)$, and $[h, g] \notin \gamma_{k+1}(G)$. Note that $k \geq 1$. By Lemma 2.11 there is a homomorphism $\psi:\langle g\rangle \rightarrow \gamma_{k}(G) / \gamma_{k+1}(G)$, but since the torsion of $\gamma_{k}(G) / \gamma_{k+1}(G)$ is trivial, $\psi$ is injective. Since $g^{n} \in H$, it follows that $\left[h, g^{n}\right]=\psi\left(g^{n}\right)=1$. But $g^{n} \neq 1$, because $g \neq 1$, and $G$ is torsion-free. It contradicts injectivity of $\psi$.

2.4. Isomorphic finitely induced representations. We have the following criterion of isomorphism of irreducible monomial representations.

Definition 2.12. We say that pairs $\left(H_{1}, \chi_{1}\right)$ and $\left(H_{2}, \chi_{2}\right)$ are equivalent if $\operatorname{ind}_{H_{1}}^{G}\left(\chi_{1}\right) \simeq \operatorname{ind}_{H_{2}}^{G}\left(\chi_{2}\right)$. We denote it as follows: $\left(H_{1}, \chi_{1}\right) \sim\left(H_{2}, \chi_{2}\right)$.

Proposition 2.13. [2, Proposition 4.10] Let $\left(H_{1}, \chi_{1}\right)$ and $\left(H_{2}, \chi_{2}\right)$ be two irreducible weight pairs. Then they are equivalent if and only if there exists an element $g \in G$ such that $\left(H_{2}^{*}\right)^{g}=H_{1}^{*}$ and $\left.\chi_{1}\right|_{H_{2}^{g} \cap H_{1}}=\left.\chi_{2}^{g}\right|_{H_{2}^{g} \cap H_{1}}$. 
Let $Y$ be the set of irreducible weight pairs. Let $\Sigma$ be the set of all subgroups $H \subset G$ for which there exists a character $\chi: H \rightarrow \mathbb{C}^{*}$ such that a pair $(H, \chi)$ belongs to $Y$. One has a natural surjective map $Y \rightarrow \Sigma$.

Let us denote by $\sim_{f}$ the equivalence 2.12, restricted on fibers of the map $Y \rightarrow \Sigma$. We denote the quotient by this equivalence by $Z_{H}=Y_{H} / \sim_{f}$, where $Y_{H}$ is a fiber of $Y$ over a subgroup $H \in \Sigma$. We denote by $Z$ the bundle over $\Sigma$ with fibres $Z_{H}$ over a subgroup $H \in \Sigma$.

Let us denote by $W$ the set of equivalence classes of irreducible weight pairs such that $\left(H_{1}, \chi_{1}\right)$ and $\left(H_{2}, \chi_{2}\right)$ belong to the same class if the pairs are equivalent, and $H_{1}^{*}=H_{2}^{*}$. Let us denote this equivalence by $\sim_{*}$. This equivalence relation on $Y$ naturally descends to the set of subgroups $\Sigma$. Subgroups $H_{1}$, $H_{2} \in \Sigma$ belong to the same equivalence class if $H_{1}^{*}=H_{2}^{*}$. Let us denote the quotient og $\Sigma$ by this equivalence by $\Theta$. There is a natural surjective map $W \rightarrow \Theta$.

Corollary 2.14. We have the following commutative diagram:

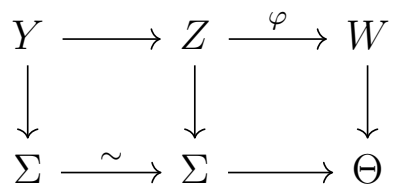

Definition 2.15. A weight pair $\left(H^{\prime}, \chi^{\prime}\right)$ extends a weight pair $(H, \chi)$ if $H$ is a subgroup of $H^{\prime}$ and $\left.\chi^{\prime}\right|_{H}=\chi$.

Proposition 2.16. Let $G$ be a finitely generated nilpotent group. Then for every representative of equivalent weight pairs $(H, \chi) \in W$ the set of equivalence classes of weight pairs $\varphi^{-1}((H, \chi))$ in $Z$ is finite.

Proof. Let $(H, \chi)$ be an element of $W$. Let us prove that there are finitely many equivalence classes of weight pairs $\left(H^{\prime}, \chi^{\prime}\right) \in Z$ such that $\left(H^{\prime}, \chi^{\prime}\right) \sim(H, \chi)$ and $H^{*}=H^{*}$.

Since $G$ is finitely generated nilpotent group, $G$ is Noetherian. Then every subgroup $H \subset G$ is finitely generated. Let $H=\left\langle h_{1}, \ldots, h_{k}\right\rangle$. Since $H^{*}=H^{*}$, there exist certain $n_{i} \in \mathbb{N}, m_{i} \in \mathbb{Z}$ such that $H^{\prime}=\left\langle h_{1}^{\frac{n_{1}}{m_{1}}}, \ldots, h_{k}^{\frac{n_{k}}{m_{k}}}\right\rangle$. For any element $h \in H$ there are finitely many $g \in G$ such that $g^{r}=h$ for some integer $r$, since for any subgroup $H$ its index in $H^{*}$ is finite. Thus, for $h_{i}, 1 \leq i \leq k$, there are finitely many integer valued tuples $\left(m_{1}^{j}, \ldots, m_{k}^{j}\right)$ such that $h_{i}^{\frac{1}{m_{1}^{j}}} \in G$ for all indices $i, j$. Let us fix a tuple $\left(m_{1}^{j}, \ldots, m_{k}^{j}\right)$ from this finite set. Let us assume that there exist infinitely many natural valued tuples $\left(n_{1}^{j}, \ldots, n_{k}^{j}\right)$ 
such that a subgroup $H^{\prime}=\left\langle h_{1}^{\frac{n_{1}}{m_{1}}}, \ldots, h_{k}^{\frac{n_{k}}{m_{k}}}\right\rangle$ belongs to $\Sigma$, and there exists a character $\chi^{\prime}$ of $H^{\prime}$ that $\left(H^{\prime}, \chi^{\prime}\right) \in Z$ and $\left(H^{\prime}, \chi^{\prime}\right) \sim(H, \chi)$.

We call two tuples $\left(n_{1}^{i}, \ldots, n_{k}^{i}\right)$ and $\left(n_{1}^{j}, \ldots, n_{k}^{j}\right)$ comparable if $n_{l}^{i} \leq n_{l}^{j}$ for all indices $1 \leq l \leq k$, or $n_{l}^{i} \geq n_{l}^{j}$ for all indices $1 \leq l \leq k$. We claim that in an infinite set of natural valued tuples there always exist two comparable tuples. Indeed, for any given tuple $\left(n_{1}^{1}, \ldots, n_{k}^{1}\right)$ let us find a tuple $\left(n_{1}^{2}, \ldots, n_{k}^{2}\right)$ which is incomparable with $\left(n_{1}^{1}, \ldots, n_{k}^{1}\right)$. It means that there exists at least one (but not all) index $1 \leq l \leq k$ such that $n_{l}^{2}<n_{l}^{1}$ and other indices $1 \leq r \leq k$ that $n_{r}^{2} \geq$ $n_{r}^{1}$. Let us construct the next tuple which is incomparable with the first one and the second one. Then there exists an index $1 \leq s \leq k$ which correspond to the value of the third tuple which is strictly smaller than corresponding value of the second tuple. This index $s$ either coincides with $l$ or does not. The set of different natural numbers $n_{1}, \ldots, n_{k}$ such that $n_{1} \leq n_{1}^{1}, \ldots, n_{k} \leq n_{k}^{1}$ is finite. If $l \neq s$ then there exists an index with strictly smaller value than of the first tuple, and we choose it from the finite set of values. If $l=s$ and the corresponding value of the third tuple $\left(n_{1}^{3}, \ldots, n_{k}^{3}\right)$ coincide in $l=s$ with the value of the second tuple, that is $n_{l}^{1}<n_{l}^{3}=n_{2}^{l}$, then we proceed by induction on $k$. Since for this case the value in $l=s$ is fixed, we are now dealing with tuples of $(k-1)$ size. It is easy to check that for $k=2$ the claim is true : there is no infinite set of incomparable natural valued tuples of the form $\left(n_{1}^{j}, n_{2}^{j}\right)$, hence the base of induction is valid.

Thus, in our infinite set of tuples $\left(n_{1}^{j}, \ldots, n_{k}^{j}\right)$ which correspond to subgroups $\left\langle h_{1}^{\frac{n_{1}^{j}}{m_{1}}}, \ldots, h_{k}^{\frac{n_{k}^{j}}{m_{k}}}\right\rangle$, there exist two comparable tuples $\left(n_{1}^{j}, \ldots, n_{k}^{j}\right)$ and $\left(n_{1}^{i}, \ldots, n_{k}^{i}\right)$. Let us denote them by $H_{i}=\left\langle h_{1}^{\frac{n_{1}^{i}}{m_{1}}}, \ldots, h_{k}^{\frac{n_{k}^{i}}{m_{k}}}\right\rangle$ and $H_{j}=$ $\left\langle h_{1}^{\frac{n_{1}^{j}}{m_{1}}}, \ldots, h_{k}^{\frac{n_{k}^{j}}{m_{k}}}\right\rangle$. Then there exist characters $\chi_{i}$ of $H_{i}$ and $\chi_{j}$ of $H_{j}$ such that weight pairs $\left(H_{i}, \chi_{i}\right)$ and $\left(H_{j}, \chi_{j}\right)$ are in $Z$, and $\left(H_{i}, \chi_{i}\right) \sim\left(H_{j}, \chi_{j}\right)$. Without loss of generality let $\left(n_{1}^{i}, \ldots, n_{k}^{i}\right)<\left(n_{1}^{j}, \ldots, n_{k}^{j}\right)$, then the weight pair $\left(H_{j}, \chi_{j}\right)$ extends the weight pair $\left(H_{i}, \chi_{i}\right)$. It contradicts the fact that both of them are irreducible weight pairs. Then the set of tuples is finite, and correspondingly, the set of weight pairs $\left(H^{\prime}, \chi^{\prime}\right) \in Z$ such that $\left(H^{\prime}, \chi^{\prime}\right) \sim(H, \chi)$ and $H^{\prime *}=H^{*}$ is also finite.

For a given weight pair $(H, \chi) \in Y$, if the quotient $G / N_{G}\left(H^{*}\right)$ is non-trivial, then it is infinite. For any element $g \in G \backslash N_{G}\left(H^{*}\right)$ the weight pair $\left(H^{g}, \chi^{g}\right)$ is irreducible and equivalent to the pair $(H, \chi)$. Since conjugation on irreducible weight pairs by elements of $G$ commutes with the mapping to a $\sim_{*}$-equivalent 
weight pair, we can take the consequent quotients of $Y$ by these equivalences. We denote by $X$ the quotient of $Y$ by this equivalences.

The latter equivalence relation naturally descends to the set of subgroups $\Sigma$. Subgroups $H_{1}, H_{2} \in \Sigma$ belong to the same equivalence class if there exists an element $g \in G$ such that $\left(H_{1}^{*}\right)^{g}=H_{2}^{*}$. We denote by $\Xi$ the quotient by this equivalence of $\Theta$. There is a natural surjective map $X \rightarrow \Xi$.

Thus, we have the following commutative diagram:

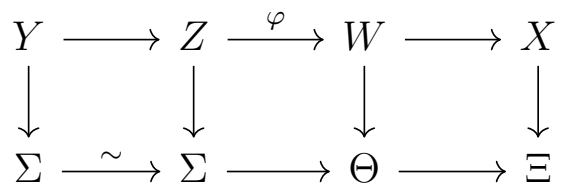

\subsection{Ranks of finitely generated nilpotent groups.}

Definition 2.17. Let $G$ be a finitely generated nilpotent group. For a given subgroup $H \subset G$ we inductively define $\operatorname{rk}_{1}(G):=\operatorname{rk}(H / H \cap[G, G])$, $\operatorname{rk}_{i+1}(H):=\operatorname{rk}\left(\left(H \cap \gamma_{i}(G)\right) /\left(H \cap \gamma_{i+1}(G)\right)\right)$.

Remark 2.18. If $\left(H_{1}, \chi_{1}\right)$ and $\left(H_{2}, \chi_{2}\right)$ are equivalent irreducible weight pairs, then $\operatorname{rk}_{i}\left(H_{1}\right)=\operatorname{rk}_{i}\left(H_{2}\right)$ for all $i$.

Proof. If $H_{1}^{*}=H_{2}^{*}$ then the ranks are clearly equal since ranks do not change if one restricts to a subgroup of a finite index.

Let $\left(H_{1}^{*}\right)^{g}=H_{2}^{*}$ for some non-trivial $g \in G$. Let $h_{1}, \ldots, h_{n}$ generate the quotient $\left(H_{1} \cap \gamma_{i}(G)\right) /\left(H_{1} \cap \gamma_{i+1}(G)\right)$. Since $\left(\left\langle h_{1}^{g}, \ldots, h_{n}^{g}\right\rangle \cap \gamma_{i}(G)\right) /\left(H_{1} \cap\right.$ $\left.\gamma_{i+1}(G)\right)$ coincides with the quotient $\left(\left\langle h_{1}, \ldots, h_{n}\right\rangle \cap \gamma_{i}(G)\right) /\left(H_{1} \cap \gamma_{i+1}(G)\right)$, we have $\operatorname{rk}_{i}\left(H_{1}\right)=\operatorname{rk}_{i}\left(H_{2}\right)$ for all $i$.

\section{Classification of IRREDUCiBle Weight PAIRS}

Let $G$ be the group of upper triangular matrices of the fourth order with integer entries. We will classify all irreducible weight pairs $(H, \chi)$ such that $H \subset G$.

Proposition 3.1. [(i)] The ranks of subgroups of the group $G$ can be the following: $(0,0),(0,1),(0,2),(1,0),(1,1),(1,2),(2,0),(2,1),(2,2)$ and $(3,2)$.

[(ii)] If $H \in \Sigma$, then ranks of $H$ can be the following: $(1,1),(2,0),(2,1)$, $(1,2),(2,2)$ and $(3,2)$.

Proof. [(i)] If $\operatorname{rk}_{1}(H)=3$, then there are two generators of $H$ in $H \cap$ $[G, G] / Z(G)$, so $\operatorname{rk}_{2}(H)=2$. Thus, cases $(3,0)$ and $(3,1)$ are not possible. 
[(ii)] It is easy to check that for any $g \in G$ the centralizer $C_{G}(g) \supsetneq\langle g, Z(G)\rangle$. For an irreducible weight pair $(H, \chi)$ we have $S(H, \chi)=H$, and by Remark 2.6 for $g \in H$ a subgroup $\langle g, Z(G)\rangle \subsetneq H$. Then there are always more than two generators in $H$. Thus, the cases $(0,0)$, $(0,1)$, and $(1,0)$ are not possible. The case of $(0,2)$ ranks is also not possible since the centralizer $C_{G}(H)$ coincides with the following subgroup of $G$ :

$$
\left(\begin{array}{llll}
1 & 0 & \mathbb{Z} & \mathbb{Z} \\
0 & 1 & \mathbb{Z} & \mathbb{Z} \\
0 & 0 & 1 & 0 \\
0 & 0 & 0 & 1
\end{array}\right)
$$

which is bigger than $H$. Then there exists a weight pair $\left(H^{\prime}, \chi^{\prime}\right)$ which extends the pair $(H, \chi)$. It contradicts the condition $S(H, \chi)=H$.

Let us denote by $\Sigma_{r_{1}, r_{2}}$ the restriction of $\Sigma$ to the set of subgroups $H \subset G$ such that $\operatorname{rk}_{1}(H)=r_{1}, \operatorname{rk}_{2}(H)=r_{2}$. The set $\Sigma$ is a disjoint union of sets $\Sigma_{r_{1}, r_{2}}$. Let us denote corresponding bundles $Y, Z$ restricted to $\Sigma_{r_{1}, r_{2}}$ by $Y_{r_{1}, r_{2}}$, $Z_{r_{1}, r_{2}}$, and similarly the bundle $X_{r_{1}, r_{2}} \rightarrow \Xi_{r_{1}, r_{2}}$.

In this subsection we describe consecutively the set $\Sigma_{r_{1}, r_{2}}$, bundles $Y_{r_{1}, r_{2}}$, $Z_{r_{1}, r_{2}}$, and $X_{r_{1}, r_{2}} \rightarrow \Xi_{r_{1}, r_{2}}$ for all possible ranks of subgroups of the set $\Sigma$ for the group $G$.

We denote by $\mu_{\infty}$ the union of groups of roots from unity.

$$
\text { 4. The CASE OF } \operatorname{rk}_{1}(H)=1, \operatorname{rk}_{2}(H)=1 \text {. }
$$

Let us define sets $S, S_{1}, S_{2}, S_{3}, S_{4}, N_{1}$, and $N_{2}$.

$$
\begin{gathered}
S=\left\{(a, d, f, b, e) \in \mathbb{Z}^{5} \mid \operatorname{GCD}\left(\frac{f b-a e}{n}, a, d, f\right)=1, \quad n=\operatorname{GCD}(a, f)\right\}, \\
S_{1}=\left\{(a, d, f, b, e) \in \mathbb{Z}^{5} \mid a \neq 0, d \neq 0, f \neq 0\right\} \cap S, \\
S_{2}=\left\{(a, d, f, b, e) \in \mathbb{Z}^{5} \mid a=0, d \neq 0, f \neq 0\right\} \cap S, \\
S_{3}=\left\{(a, d, f, b, e) \in \mathbb{Z}^{5} \mid a \neq 0, d \neq 0, f=0\right\} \cap S, \\
S_{4}=\left\{(a, d, f, b, e) \in \mathbb{Z}^{5} \mid a \neq 0, d=0, f \neq 0\right\} \cap S, \\
N_{1}=\left\{(a, d, f, b, e) \in \mathbb{Z}^{5} \mid \begin{array}{c}
a \neq 0, d=0, f=0, b=1\} \cap S, \\
9
\end{array}\right.
\end{gathered}
$$




$$
N_{2}=\left\{(a, d, f, b, e) \in \mathbb{Z}^{5} \mid a=0, d=0, f \neq 0, e=1\right\} \cap S .
$$

Proposition 4.1. There is a canonical bijection $\phi$ from $S_{1} \cup S_{2} \cup S_{3} \cup S_{4} \cup$ $N_{1} \cup N_{2}$ to $\Sigma_{1,1}$. It maps a tuple $(a, d, f, b, e)$ to a subgroup $H$, generated by the following matrices

$$
h_{1}=\left(\begin{array}{cccc}
1 & a & b & 0 \\
0 & 1 & d & e \\
0 & 0 & 1 & f \\
0 & 0 & 0 & 1
\end{array}\right), \quad h_{2}=\left(\begin{array}{cccc}
1 & 0 & \frac{a}{n} & 0 \\
0 & 1 & 0 & \frac{f}{n} \\
0 & 0 & 1 & 0 \\
0 & 0 & 0 & 1
\end{array}\right), \quad C=\left(\begin{array}{cccc}
1 & 0 & 0 & 1 \\
0 & 1 & 0 & 0 \\
0 & 0 & 1 & 0 \\
0 & 0 & 0 & 1
\end{array}\right),
$$

where $n=\operatorname{GCD}(a, f)$. Moreover, for every $H \in \Sigma_{1,1}$, a subgroup $H$ is abelian.

We can extend the bijection $\phi$ to the map from

$$
\left(\mathbb{C}^{*}\right)^{2} \times\left\{\mathbb{C}^{*} \backslash \mu_{\infty}\right\}=\left\{t, z \in\left(\mathbb{C}^{*}\right)^{2}, \lambda \notin \mu_{\infty}\right\}
$$

to $Y_{1,1 ; H}$, which is defined as follows:

$$
t=\chi\left(h_{1}\right), \quad z=\chi\left(h_{2}\right), \quad \lambda=\chi(C) .
$$

Proof. The proof goes as follows. First, we prove that if $H \in \Sigma_{1,1}$, then a subgroup $H$ is abelian, and it is generated by $h_{1}, h_{2}, C$ in the form given in Proposition 4.1. Then we obtain conditions for generators $h_{1}, h_{2}$ that the corresponding subgroup $H$ can form an irreducible weight pair with some character $\chi$. Then we study characters $\chi_{1}, \chi_{2}: H \rightarrow \mathbb{C}^{*}$ which correspond to equivalent irreducible weight pairs $\left(H, \chi_{1}\right)$ and $\left(H, \chi_{2}\right)$. Finally, we obtain conditions for a character $\chi$ that $(H, \chi)$ is an irreducible weight pair.

Since $\operatorname{rk}_{1}(H)=\operatorname{rk}_{2}(H)=1$, we have one generator $h_{1}$ such that $\left\langle h_{1}\right\rangle /([G, G] \cap H)$ is not trivial. Let us denote by $h_{2}$ a generator such that $\left\langle h_{2}\right\rangle /([G, G] \cap H)$ is trivial and $\left(\left\langle h_{2}\right\rangle \cap[G, G]\right) / Z(G)$ is not trivial. By Remark 2.6 the center $Z(G)$ is contained in $H$, hence we can choose generators $h_{1}$ and $h_{2}$ such that $\left\langle h_{1}\right\rangle \cap Z(G)$ and $\left\langle h_{2}\right\rangle \cap Z(G)$ are trivial.

If the commutator $\left[h_{1}, h_{2}\right]$, which is contained in the center of $G$, is not trivial, then $\chi\left(\left[h_{1}, h_{2}\right]\right)=\chi(C)^{N}=1$ for some integer $N$. But if $\chi(C)$ is a root of unity, then $\operatorname{rk}_{2}\left(C_{G}(H)\right)=2$, since in this case both elements

$$
\left(\begin{array}{cccc}
1 & 0 & k_{1} & 0 \\
0 & 1 & 0 & 0 \\
0 & 0 & 1 & 0 \\
0 & 0 & 0 & 1
\end{array}\right), \quad\left(\begin{array}{cccc}
1 & 0 & 0 & 0 \\
0 & 1 & 0 & k_{2} \\
0 & 0 & 1 & 0 \\
0 & 0 & 0 & 1
\end{array}\right)
$$

are contained in $H$ for some integers $k_{1}$ and $k_{2}$. By Remark 2.6 the centralizer $C_{G}(H) \subset H$, thus $\chi(C)$ is not a root of unity.

Hence, generators $h_{1}, h_{2}$ commute, and $H$ is abelian. Then it follows from Lemma 2.10 that $H=H^{*}$. 
Put

$$
h_{1}=\left(\begin{array}{llll}
1 & a & b & 0 \\
0 & 1 & d & e \\
0 & 0 & 1 & f \\
0 & 0 & 0 & 1
\end{array}\right)
$$

If $a=f=0$, then $\operatorname{rk}_{2}\left(C_{G}(H)\right)=2$, but the centralizer $C_{G}(H) \subset H$ by Remark 2.6. Then either $a$ or $f$ are non-zero.

Since generators $h_{1}, h_{2}$ commute, we obtain that $h_{2}^{k}$ is equal to

$$
\left(\begin{array}{llll}
1 & 0 & a & 0 \\
0 & 1 & 0 & f \\
0 & 0 & 1 & 0 \\
0 & 0 & 0 & 1
\end{array}\right)
$$

for some integer $k$. If $a$ and $f$ are coprime then $h_{2}$ coincides with this element. If not, then since a subgroup $H$ is isolated, it has to contain all its roots. Hence

$$
h_{2}=\left(\begin{array}{cccc}
1 & 0 & \frac{a}{n} & 0 \\
0 & 1 & 0 & \frac{f}{n} \\
0 & 0 & 1 & 0 \\
0 & 0 & 0 & 1
\end{array}\right)
$$

where $n=\operatorname{GCD}(a, f)$. Let us denote by $\left(a^{\prime}, f^{\prime}\right)$ the proportional coprime tuple $\left(\frac{a}{n}, \frac{f}{n}\right)$.

Now we will obtain conditions for a subgroup $H$ to be an isolated subgroup. In order to do that we will study tuples $(a, d, f, b, e)$ which correspond to generators of subgroups $H$ such that $H \subset H^{*}$.

Let $g \in H^{*} \backslash H$. That means that there are such natural $r_{1}>1$ and integer $r_{2}, r_{3}$ that $g^{r_{1}}=h_{1}^{r_{2}} h_{2}^{r_{3}}$. Let

$$
g=\left(\begin{array}{cccc}
1 & \tilde{a} & \tilde{b} & 0 \\
0 & 1 & \tilde{d} & \tilde{e} \\
0 & 0 & 1 & \tilde{f} \\
0 & 0 & 0 & 1
\end{array}\right)
$$

That means that

$$
\left(\begin{array}{cccc}
1 & r_{1} \tilde{a} & r_{1} \tilde{b}+\frac{r_{1}\left(r_{1}-1\right)}{2} \tilde{a} \tilde{d} & 0 \\
0 & 1 & r_{1} \tilde{d} & r_{1} \tilde{e}+\frac{r_{1}\left(r_{1}-1\right)}{2} \tilde{f} \tilde{d} \\
0 & 0 & 1 & r_{1} f \\
0 & 0 & 0 & 1
\end{array}\right)=
$$




$$
\left(\begin{array}{cccc}
1 & r_{2} a & r_{2} b+\frac{r_{2}\left(r_{2}-1\right)}{2} a d & 0 \\
0 & 1 & r_{2} d & r_{2} e+\frac{r_{2}\left(r_{2}-1\right)}{2} f d \\
0 & 0 & 1 & r_{2} f \\
0 & 0 & 0 & 1
\end{array}\right) \times\left(\begin{array}{cccc}
1 & 0 & r_{3} a^{\prime} & 0 \\
0 & 1 & 0 & r_{3} f^{\prime} \\
0 & 0 & 1 & 0 \\
0 & 0 & 0 & 1
\end{array}\right)
$$

It implies that

$$
\left(\begin{array}{c}
r_{1} \tilde{a} \\
r_{1} \tilde{d} \\
r_{1} \tilde{f}
\end{array}\right)=\left(\begin{array}{c}
r_{2} a \\
r_{2} d \\
r_{2} f
\end{array}\right) \text { and }\left(\begin{array}{c}
r_{1} \tilde{a} \\
r_{1} \tilde{d} \\
r_{1} \tilde{f}
\end{array}\right)=l r_{1}\left(\begin{array}{l}
a^{\prime \prime} \\
d^{\prime \prime} \\
f^{\prime \prime}
\end{array}\right),
$$

where $\left(a^{\prime \prime}, d^{\prime \prime}, f^{\prime \prime}\right)$ is a coprime tuple which is proportional to $(a, d, f)$.

Then $r_{1} l=r_{2} n$ for some integer $l$. Let us notice that if $n=1$ then it is easy to observe that $g$ is an element of $H$.

If $\operatorname{GCD}(l, n)$ is greater than 1 , then we may divide the equality $r_{1} l=r_{2} n$ by $\operatorname{GCD}(l, n)$ and obtain the new equality $r_{1} l^{\prime}=r_{2} n^{\prime}$ with $\operatorname{GCD}\left(l^{\prime}, n^{\prime}\right)=1$. After that, we can replace a tuple $(l, n)$ with a proportional coprime tuple $\left(l^{\prime}, n^{\prime}\right)$. Since modulo this replacement it does not change the proof, we proceed with the case when $\operatorname{GCD}(l, n)=1$.

We have $r_{1}=n r$ and $r_{2}=l r$ for some integer $r$.

From $g^{r_{1}}=h_{1}^{r_{2}} h_{2}^{r_{3}}$ we obtain

$$
\begin{aligned}
& r_{1} \tilde{b}+\frac{r_{1}\left(r_{1}-1\right)}{2} l^{2} a^{\prime \prime} d^{\prime \prime}=r_{2} b+\frac{r_{2}\left(r_{2}-1\right)}{2} n^{2} a^{\prime \prime} d^{\prime \prime}+r_{3} a^{\prime}, \\
& r_{1} \tilde{e}+\frac{r_{1}\left(r_{1}-1\right)}{2} l^{2} f^{\prime \prime} d^{\prime \prime}=r_{2} e+\frac{r_{2}\left(r_{2}-1\right)}{2} n^{2} f^{\prime \prime} d^{\prime \prime}+r_{3} f^{\prime} .
\end{aligned}
$$

Then

$$
\begin{aligned}
& r_{1} \tilde{b}-r_{2} b=\left(\frac{r_{2}\left(r_{2}-1\right)}{2} n^{2} k d^{\prime \prime}-\frac{r_{1}\left(r_{1}-1\right)}{2} l^{2} k d^{\prime \prime}+r_{3}\right) a^{\prime}, \\
& r_{1} \tilde{e}-r_{2} e=\left(\frac{r_{2}\left(r_{2}-1\right)}{2} n^{2} k d^{\prime \prime}-\frac{r_{1}\left(r_{1}-1\right)}{2} l^{2} k d^{\prime \prime}+r_{3}\right) f^{\prime} .
\end{aligned}
$$

Let us denote the expression $\left(\frac{r_{2}\left(r_{2}-1\right)}{2} n^{2} k d^{\prime \prime}-\frac{r_{1}\left(r_{1}-1\right)}{2} l^{2} k d^{\prime \prime}+r_{3}\right)$ by $M$. Then $M$ can be an arbitrary integer since $r_{3}$ can be an arbitrary integer. Now we need to obtain the condition for the system of equations

$$
\begin{aligned}
& r(n \tilde{b}-l b)=M a^{\prime}, \\
& r(n \tilde{e}-l e)=M f^{\prime} .
\end{aligned}
$$

to be solvable in $\tilde{b}, \tilde{e} \in \mathbb{Z}^{2}$, where integers $M, r$, and $l$ are coprime with $n$, and integers $b, e, n, a^{\prime}, f^{\prime}$ are fixed. 
The system is solvable if and only if

$$
l\left(b f^{\prime}-e a^{\prime}\right)=n\left(\tilde{b} f^{\prime}-\tilde{e} a^{\prime}\right) .
$$

Since $n$ and $l$ are coprime, the condition above is equivalent to the following one: $\left(b f^{\prime}-e a^{\prime}\right)$ is divisible by $n$. Then $\left(\tilde{b} f^{\prime}-\tilde{e} a^{\prime}\right)=l m$ for some integer $m$ and since $a^{\prime}$ and $f^{\prime}$ are coprime, there always exist integers $\tilde{b}$ and $\tilde{e}$ which satisfy the equation.

Finally, we can see that a subgroup $H$ is isolated if and only if $H \in \phi(S)$.

Now for every $H \in \Sigma_{1,1}$, we will describe the fiber $Y_{1,1 ; H}$. We need to check that $Y_{1,1 ; H}$ parametrizes all characters $\chi$ of $H$ which correspond to irreducible weight pairs $(H, \chi)$.

(i). If $H \in \Sigma_{1,1} \backslash\left(\phi\left(N_{1}\right) \cup \phi\left(N_{2}\right) \cup \phi\left(S_{2}\right) \cup \phi\left(S_{3}\right)\right)$ then the quotient $N_{G}(H) / H$ is generated by

$$
g_{1}=\left(\begin{array}{cccc}
1 & a^{\prime} & 0 & 0 \\
0 & 1 & 0 & 0 \\
0 & 0 & 1 & -f^{\prime} \\
0 & 0 & 0 & 1
\end{array}\right), \quad g_{2}=\left(\begin{array}{cccc}
1 & 0 & 0 & 0 \\
0 & 1 & 0 & 1 \\
0 & 0 & 1 & 0 \\
0 & 0 & 0 & 1
\end{array}\right)
$$

The action of $g_{1}$ and $g_{2}$ on a character $\chi$ is as follows:

$$
\begin{gathered}
\chi^{g_{1}}\left(h_{1}\right)=\chi\left(h_{1}\right) \chi\left(h_{2}\right)^{d} \lambda^{a^{\prime} e+f^{\prime} b+a^{\prime} f^{\prime} d}, \quad \chi^{g_{1}}\left(h_{2}\right)=\chi\left(h_{2}\right) \lambda^{2 a^{\prime} f^{\prime}}, \\
\text { and } \\
\chi^{g_{2}}\left(h_{1}\right)=\chi\left(h_{1}\right) \lambda^{-a}, \quad \chi^{g_{2}}\left(h_{2}\right)=\chi\left(h_{2}\right) .
\end{gathered}
$$

We can see that if $\chi(C)=\lambda$ is not a root of unity, then the action above is free, which means that $S(H, \chi)=H$ for a corresponding weight pair $(H, \chi)$.

If $H \in \phi\left(S_{2}\right)$, then the generator $g_{2}$ is replaced by

$$
g_{2}=\left(\begin{array}{llll}
1 & 0 & 1 & 0 \\
0 & 1 & 0 & 0 \\
0 & 0 & 1 & 0 \\
0 & 0 & 0 & 1
\end{array}\right)
$$

The action is as follows:

$$
\chi^{g_{1}}\left(h_{1}\right)=\chi\left(h_{1}\right) \chi\left(h_{2}\right)^{d} \lambda^{f^{\prime} b}, \quad \chi^{g_{1}}\left(h_{2}\right)=\chi\left(h_{2}\right),
$$




$$
\chi^{g_{2}}\left(h_{1}\right)=\chi\left(h_{1}\right) \lambda^{f}, \quad \chi^{g_{2}}\left(h_{2}\right)=\chi\left(h_{2}\right) .
$$

Again, we can see that if $\chi(C)=\lambda$ is not a root of unity, then the action above is free, which means that $S(H, \chi)=H$.

(ii). If $H \in\left(\phi\left(N_{1}\right) \cup \phi\left(N_{2}\right)\right)$, then it is easy to check that $N_{G}(H)=G$. Without loss of generality let us consider the case of a subgroup $H$ with $f=$ $d=0$. The other one with $a=d=0$ is treated similarly. Since $H$ is isolated, its generators $h_{1}$ and $h_{2}$ may be chosen as follows:

$$
h_{1}=\left(\begin{array}{llll}
1 & a & 0 & 0 \\
0 & 1 & 0 & e \\
0 & 0 & 1 & 0 \\
0 & 0 & 0 & 1
\end{array}\right), \quad h_{2}=\left(\begin{array}{llll}
1 & 0 & 1 & 0 \\
0 & 1 & 0 & 0 \\
0 & 0 & 1 & 0 \\
0 & 0 & 0 & 1
\end{array}\right)
$$

Then the quotient $N_{G}(H) / H$ is generated by

$$
\begin{gathered}
g_{1}=\left(\begin{array}{llll}
1 & 0 & 0 & 0 \\
0 & 1 & 1 & 0 \\
0 & 0 & 1 & 0 \\
0 & 0 & 0 & 1
\end{array}\right), \quad g_{2}=\left(\begin{array}{llll}
1 & 0 & 0 & 0 \\
0 & 1 & 0 & 1 \\
0 & 0 & 1 & 0 \\
0 & 0 & 0 & 1
\end{array}\right) \\
\text { and } \\
g_{3}=\left(\begin{array}{llll}
1 & 0 & 0 & 0 \\
0 & 1 & 0 & 0 \\
0 & 0 & 1 & 1 \\
0 & 0 & 0 & 1
\end{array}\right) .
\end{gathered}
$$

The action of $g_{1}, g_{2}$ and $g_{3}$ on characters is as follows:

$$
\begin{aligned}
& \chi^{g_{1}}\left(h_{1}\right)=\chi\left(h_{1}\right) \chi\left(h_{2}\right)^{-a}, \quad \chi^{g_{1}}\left(h_{2}\right)=\chi\left(h_{2}\right), \\
& \chi^{g_{2}}\left(h_{1}\right)=\chi\left(h_{1}\right) \chi(C)^{-a}, \quad \chi^{g_{2}}\left(h_{2}\right)=\chi\left(h_{2}\right), \\
& \chi^{g_{3}}\left(h_{1}\right)=\chi\left(h_{1}\right), \quad \chi^{g_{3}}\left(h_{2}\right)=\chi\left(h_{2}\right) \chi(C)^{-1} .
\end{aligned}
$$

Again, we can see that if $\chi(C)=\lambda$ is not a root of unity, then the action above is free, which means that $S(H, \chi)=H$ and corresponding representations are irreducible.

Let us consider the following action: $z \rightarrow z \lambda^{n}, n \in \mathbb{Z}$.

If $\lambda$ is a root of unity, then the quotient of $z \in \mathbb{C}^{*}$ by this action is conformally equivalent to $\mathbb{C}^{*}$.

In a case that $\lambda$ is not in $S^{1}$, then the quotient by this action is an elliptic curve, which we denote by $E_{\lambda}=\left\langle\mathbb{C}^{*} / \lambda^{n}, n \in \mathbb{Z}\right\rangle$.

In a case that $\lambda \in S^{1} \backslash \mu_{\infty}$, we denote the corresponding quotient by $P_{\lambda}$ (not separable). 
Let both $\lambda$ and $z$ be not roots of unity. We denote by $T_{z, \lambda}=$ $\left\langle\mathbb{C}^{*} / \lambda^{n_{1}} z^{n_{2}}, n_{1}, n_{2} \in \mathbb{Z}^{2}\right\rangle$ (not separable).

Let us denote by $Z_{1,1 ; H}^{(3)}=\left\{\left\{t, z \in\left(\mathbb{C}^{*}\right)^{2}, \lambda \notin \mu_{\infty}\right\} / \sim\right\}$, by $Z_{1,1 ; H}^{(2)}=\{\{z \in$ $\left.\left.\mathbb{C}^{*}, \lambda \notin \mu_{\infty}\right\} / \sim\right\}$ and by $Z_{1,1 ; H}^{(1)}=\left\{\lambda \notin \mu_{\infty}\right\}$, where $\sim$ is defined in 2.12.

Corollary 4.5. If $H \in \phi\left(S_{1}\right)$, then the fiber $Z_{1,1 ; H}$ of $Z_{1,1}$ over a subgroup $H$ has iterated structure of a bundle, namely:

$$
Z_{1,1 ; H}^{(3)} \rightarrow Z_{1,1 ; H}^{(2)} \rightarrow Z_{1,1 ; H}^{(1)} .
$$

We describe fibers of this bundle consecutively in coordinates $(t, z, \lambda)$.

$$
\begin{aligned}
& \left\{T_{z^{d} \lambda^{a^{\prime} e+f^{\prime} b+a^{\prime} f^{\prime} d, a},}\right\},\left\{E_{\lambda^{2 a^{\prime} f^{\prime}}} \backslash \mu_{\infty}\right\},\left\{\mathbb{C}^{*} \backslash S^{1}\right\} \cup \\
& \left\{T_{z^{d} \lambda^{a^{\prime} e+f^{\prime} b+a^{\prime} f^{\prime} d, a}}\right\},\left\{P_{\lambda^{2 a^{\prime} f^{\prime}}} \backslash \mu_{\infty}\right\},\left\{S^{1} \backslash \mu_{\infty}\right\} \cup
\end{aligned}
$$

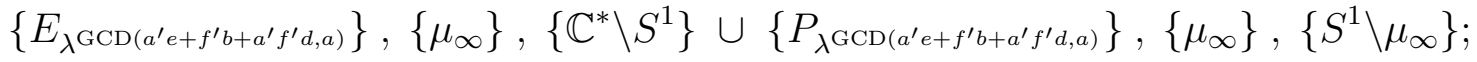

If $H \in \phi\left(S_{2}\right)$, then the fibers $Z_{1,1 ; H}^{(3)} \rightarrow Z_{1,1 ; H}^{(2)} \rightarrow Z_{1,1 ; H}^{(1)}$ over $H$ are canonically bijective to:

$$
\begin{gathered}
\left\{T_{z^{d} \lambda^{f^{\prime} b}, \lambda^{f}}\right\},\left\{\mathbb{C}^{*} \backslash S^{1}\right\},\left\{\mathbb{C}^{*} \backslash S^{1}\right\} \cup\left\{T_{z^{d} \lambda^{f^{\prime} b}, \lambda^{f}}\right\},\left\{S^{1} \backslash \mu_{\infty}\right\},\left\{\mathbb{C}^{*} \backslash S^{1}\right\} \cup \\
\left\{E_{\lambda^{\mathrm{GCD}\left(f^{\prime} b, f\right)}}\right\},\left\{\mu_{\infty}\right\},\left\{\mathbb{C}^{*} \backslash S^{1}\right\} \cup\left\{T_{z^{d} \lambda^{f^{\prime} b}, \lambda^{f}}\right\},\left\{\mathbb{C}^{*} \backslash \mu_{\infty}\right\},\left\{S^{1} \backslash \mu_{\infty}\right\} \cup \\
\left\{P_{\lambda^{\mathrm{GCD}\left(f^{\prime} b, f\right)}}\right\},\left\{\mu_{\infty}\right\},\left\{S^{1} \backslash \mu_{\infty}\right\} ;
\end{gathered}
$$

If $H \in \phi\left(S_{3}\right)$, then the fibers $Z_{1,1 ; H}^{(3)} \rightarrow Z_{1,1 ; H}^{(2)} \rightarrow Z_{1,1 ; H}^{(1)}$ over $H$ are canonically bijective to:

$$
\begin{gathered}
\left\{T_{z^{d} \lambda^{a^{\prime} e}, \lambda^{a}}\right\},\left\{\mathbb{C}^{*} \backslash S^{1}\right\},\left\{\mathbb{C}^{*} \backslash S^{1}\right\} \cup\left\{T_{z^{d} \lambda^{a^{\prime} e}, \lambda^{a}}\right\},\left\{S^{1} \backslash \mu_{\infty}\right\},\left\{\mathbb{C}^{*} \backslash S^{1}\right\} \cup \\
\left\{E_{\lambda^{\mathrm{GCD}\left(a^{\prime} e, a\right)}}\right\},\left\{\mu_{\infty}\right\},\left\{\mathbb{C}^{*} \backslash S^{1}\right\} \cup\left\{T_{z^{d} \lambda^{a^{\prime} e}, \lambda^{a}}\right\},\left\{\mathbb{C}^{*} \backslash \mu_{\infty}\right\},\left\{S^{1} \backslash \mu_{\infty}\right\} \cup \\
\left\{P_{\lambda^{\mathrm{GCD}\left(a^{\prime} e, a\right)}}\right\},\left\{\mu_{\infty}\right\},\left\{S^{1} \backslash \mu_{\infty}\right\} ;
\end{gathered}
$$

If $H \in \phi\left(S_{4}\right)$, then the fibers $Z_{1,1 ; H}^{(3)} \rightarrow Z_{1,1 ; H}^{(2)} \rightarrow Z_{1,1 ; H}^{(1)}$ over $H$ are canonically bijective to:

$\left\{E_{\lambda_{\mathrm{GCD}\left(a^{\prime} e+f^{\prime} b, a\right)}}\right\},\left\{E_{\lambda^{2 a^{\prime} f^{\prime}}}\right\},\left\{\mathbb{C}^{*} \backslash S^{1}\right\} \cup\left\{P_{\lambda_{\mathrm{GCD}\left(a^{\prime} e+f^{\prime} b, a\right)}}\right\},\left\{P_{\lambda^{2 a^{\prime} f^{\prime}}}\right\},\left\{S^{1} \backslash \mu_{\infty}\right\} ;$

If $H \in \phi\left(N_{1}\right)$, then the fibers $Z_{1,1 ; H}^{(3)} \rightarrow Z_{1,1 ; H}^{(2)} \rightarrow Z_{1,1 ; H}^{(1)}$ over $H$ are canonically bijective to:

$$
\begin{aligned}
& \left\{T_{z^{a}, \lambda^{a}}\right\},\left\{E_{\lambda} \backslash \mu_{\infty}\right\},\left\{\mathbb{C}^{*} \backslash S^{1}\right\} \cup\left\{E_{\lambda}\right\},\left\{\mu_{\infty}\right\},\left\{\mathbb{C}^{*} \backslash S^{1}\right\} \cup \\
& \left\{T_{z^{a}, \lambda^{a}}\right\},\left\{P_{\lambda} \backslash \mu_{\infty}\right\},\left\{S^{1} \backslash \mu_{\infty}\right\} \cup\left\{P_{\lambda}\right\},\left\{\mu_{\infty}\right\},\left\{S^{1} \backslash \mu_{\infty}\right\} .
\end{aligned}
$$


If $H \in \phi\left(N_{2}\right)$, then the fibers $Z_{1,1 ; H}^{(3)} \rightarrow Z_{1,1 ; H}^{(2)} \rightarrow Z_{1,1 ; H}^{(1)}$ over $H$ are canonically bijective to:

$$
\begin{aligned}
& \left\{T_{z^{f}, \lambda^{f}}\right\},\left\{E_{\lambda} \backslash \mu_{\infty}\right\},\left\{\mathbb{C}^{*} \backslash S^{1}\right\} \cup\left\{E_{\lambda}\right\},\left\{\mu_{\infty}\right\},\left\{\mathbb{C}^{*} \backslash S^{1}\right\} \cup \\
& \left\{T_{z^{f}, \lambda^{f}}\right\},\left\{P_{\lambda} \backslash \mu_{\infty}\right\},\left\{S^{1} \backslash \mu_{\infty}\right\} \cup\left\{P_{\lambda}\right\},\left\{\mu_{\infty}\right\},\left\{S^{1} \backslash \mu_{\infty}\right\} .
\end{aligned}
$$

Proof. Follows directly from formulas of the action of $N_{G}(H) / H$ on a character $\chi$ of $H$ above (equations 4.4, 4.3).

Lemma 4.6. If $H_{1} \in \Sigma_{1,1} \backslash\left(\phi\left(N_{1}\right) \cup \phi\left(N_{2}\right)\right)$, then the following subgroups $H_{2} \in \Sigma_{1,1}$ are equivalent to $H_{1}$ :

$H_{2}=\phi((a, d, f, b+\tilde{d} f, e-\tilde{d} a))$ for an arbitrary integer $\tilde{d}$.

Fibers $Z_{1,1 ; H_{1}}, Z_{1,1 ; H_{2}}$ over subgroups $H_{1}, H_{2}$ can be canonically identified.

Proof. Since $H_{1}=H_{1}^{*}$ for $H_{1} \in \Sigma_{1,1}$, as proved in Proposition 4.1, we need to consider only subgroups $H_{2}$ such that $\left(H_{2}\right)^{g}=H_{1}$ and $\left.\chi_{1}\right|_{H_{2}^{g} \cap H_{1}}=\left.\chi_{2}^{g}\right|_{H_{2}^{g} \cap H_{1}}$ for some element $g \in G$.

If $H_{1}=\phi((a, d, f, b, e)) \in \Sigma_{1,1} \backslash\left(\phi\left(N_{1}\right) \cup \phi\left(N_{2}\right)\right)$, then $H_{1}$ is not normal and the quotient $G / N_{G}\left(H_{1}\right)$ is generated by

$$
g=\left(\begin{array}{llll}
1 & 0 & 0 & 0 \\
0 & 1 & 1 & 0 \\
0 & 0 & 1 & 0 \\
0 & 0 & 0 & 1
\end{array}\right)
$$

Then it is easy to compute the parameters of conjugated subgroups: $H_{2}=\phi((a, d, f, b+\tilde{d} f, e-\tilde{d} a))$. If we denote non-central generators of $H_{2}$ by $\tilde{h}_{1}, \tilde{h}_{2}$, then characters of the subgroups are related as follows: $\chi_{2}\left(\tilde{h}_{1}\right)=\chi_{1}^{g}\left(h_{1}\right)$, $\chi_{2}\left(\tilde{h}_{2}\right)=\chi_{1}\left(h_{2}\right)$ and $\chi_{2}(C)=\chi_{1}(C)$. It gives a canonical identification of fibers $Z_{1,1 ; H_{1}}, Z_{1,1 ; H_{2}}$ over subgroups $H_{1}$ and $H_{2}$.

\section{The CASE OF $\operatorname{rk}_{1}(H)=2, \operatorname{rk}_{2}(H)=0$.}

Let us define a set

$S=\left\{\left(a, b, e, f^{\prime}, b^{\prime}, e^{\prime}\right) \in \mathbb{Z}^{6}\left|a e^{\prime}+f^{\prime} b=0, a \neq 0, f \neq 0,\right| \operatorname{GCD}(a, b, e)|=1, \quad| \operatorname{GCD}\left(f^{\prime}, b^{\prime}, e^{\prime}\right) \mid=1\right\}$.

Lemma 5.1. There is a canonical bijection $\phi$ from $S$ to $\Sigma_{2,0}$. It maps a tuple $\left(a, b, e, f^{\prime}, b^{\prime}, e^{\prime}\right)$ to a subgroup $H$, generated by the following matrices

$$
h_{1}=\left(\begin{array}{cccc}
1 & a & b & 0 \\
0 & 1 & 0 & e \\
0 & 0 & 1 & 0 \\
0 & 0 & 0 & 1
\end{array}\right), \quad h_{2}=\left(\begin{array}{cccc}
1 & 0 & b^{\prime} & 0 \\
0 & 1 & 0 & e^{\prime} \\
0 & 0 & 1 & f^{\prime} \\
0 & 0 & 0 & 1
\end{array}\right), \quad C=\left(\begin{array}{cccc}
1 & 0 & 0 & 1 \\
0 & 1 & 0 & 0 \\
0 & 0 & 1 & 0 \\
0 & 0 & 0 & 1
\end{array}\right)
$$


Moreover, for every $H \in \Sigma_{2,0}$, a subgroup $H$ is abelian.

We can extend the bijection $\phi$ to the map from

$$
\left(\mathbb{C}^{*}\right)^{2} \times\left\{\mathbb{C}^{*} \backslash \mu_{\infty}\right\}=\left\{(t, s, \lambda) \mid \lambda \notin \mu_{\infty}\right\}
$$

to $Y_{2,0 ; \phi(\alpha)}$, which is defined as follows:

$$
t=\chi\left(h_{1}\right), \quad s=\chi\left(h_{2}\right), \quad \lambda=\chi(C) .
$$

Proof. The proof goes as follows. First, we prove that if $H \in \Sigma_{2,0}$, then $H$ is generated by $h_{1}, h_{2}, C$ in the form above. After that, we prove that $H$ is abelian. After that, we obtain conditions for $h_{1}, h_{2}$ that the corresponding subgroup $H$ is isolated. Then we study characters $\chi_{1}, \chi_{2}: H \rightarrow \mathbb{C}^{*}$ which correspond to equivalent irreducible weight pairs $\left(H, \chi_{1}\right)$ and $\left(H, \chi_{2}\right)$. Finally, we obtain conditions for a character $\chi$ that $(H, \chi)$ is an irreducible weight pair.

Let us denote by $h_{1}, h_{2}$ two generators of $H / H \cap[G, G]$. Since $\operatorname{rk}_{2}(H)=0$, it follows that the commutator $\left[h_{1}, h_{2}\right]$ is in the center of $G$. If $\chi(C)$ is a root of unity, then we can extend a weight pair $(H, \chi)$ to $\left(H^{\prime}, \chi^{\prime}\right)$ with the rank $\operatorname{rk}_{2}\left(H^{\prime}\right)=2$. Hence if $\chi(C)$ is a root of unity, the pair $(H, \chi)$ is not irreducible. Then $\chi(C)$ is not a root of unity, and $h_{1}$ and $h_{2}$ commute. Then generators $h_{1}$ and $h_{2}$ have the following form:

$$
h_{1}=\left(\begin{array}{cccc}
1 & a & b & 0 \\
0 & 1 & 0 & e \\
0 & 0 & 1 & 0 \\
0 & 0 & 0 & 1
\end{array}\right), \quad h_{2}=\left(\begin{array}{cccc}
1 & 0 & b^{\prime} & 0 \\
0 & 1 & 0 & e^{\prime} \\
0 & 0 & 1 & f^{\prime} \\
0 & 0 & 0 & 1
\end{array}\right)
$$

such that $a e^{\prime}+f^{\prime} b=0$. The last condition follows from the equality $\left[h_{1}, h_{2}\right]=1$.

Since $H$ is abelian, then by Lemma 2.10 it is isolated.

The conditions for generators $h_{1}$ and $h_{2}$ that $H$ is isolated are easy to compute in this case. They are the following ones: $\operatorname{GCD}(a, b, e)=1$ and $\operatorname{GCD}\left(f^{\prime}, b^{\prime}, e^{\prime}\right)=1$.

Now for every $H \in \Sigma_{2,0}$, we need to describe the fiber $Y_{2,0 ; H}$.

For all subgroups $H \in \Sigma_{2,0}$, the quotient $N_{G}(H) / H$ is generated by

$$
g_{1}=\left(\begin{array}{llll}
1 & 0 & 1 & 0 \\
0 & 1 & 0 & 0 \\
0 & 0 & 1 & 0 \\
0 & 0 & 0 & 1
\end{array}\right), \quad g_{2}=\left(\begin{array}{cccc}
1 & 0 & 0 & 0 \\
0 & 1 & 0 & 1 \\
0 & 0 & 1 & 0 \\
0 & 0 & 0 & 1
\end{array}\right)
$$

The action of $g_{1}, g_{2}$ on a character $\chi$ is as follows:

$$
\begin{gathered}
\chi^{g_{1}}\left(h_{1}\right)=\chi\left(h_{1}\right) \lambda^{f^{\prime}}, \quad \chi^{g_{1}}\left(h_{2}\right)=\chi\left(h_{2}\right) \\
\chi^{g_{2}}\left(h_{1}\right)=\chi\left(h_{1}\right), \quad \chi^{g_{2}}\left(h_{2}\right)=\chi\left(h_{2}\right) \lambda^{-a} . \\
17
\end{gathered}
$$


We can see that the action above is free if $\chi(C)=\lambda$ is not a root of unity, which means that corresponding representations are irreducible.

Let us denote by $Z_{2,0 ; H}^{(2,1)}=\left\{\left\{t \in \mathbb{C}^{*}, \lambda \notin \mu_{\infty}\right\} / \sim\right\}$, by $Z_{2,0 ; H}^{(2,2)}=\{\{s \in$ $\left.\left.\mathbb{C}^{*}, \lambda \notin \mu_{\infty}\right\} / \sim\right\}$ and by $Z_{2,0 ; H}^{(1)}=\left\{\lambda \notin \mu_{\infty}\right\}$, where $\sim$ is defined in 2.12.

Corollary 5.3. If $H \in \Sigma_{2,0}$, then the fiber $Z_{2,0 ; H}$ of $Z_{2,0}$ over a subgroup $H$ has the following iterated structure of a bundle, namely:

$$
Z_{2,0 ; H}^{(2,1)} \rightarrow Z_{2,0 ; H}^{(1)} \quad, \quad Z_{2,0 ; H}^{(2,2)} \rightarrow Z_{2,0 ; H}^{(1)}
$$

We describe fibers of these bundles consecutively in coordinates $(t, s, \lambda)$.

$$
\left\{E_{\lambda^{f^{\prime}}}\right\},\left\{E_{\lambda^{a}}\right\},\left\{\mathbb{C}^{*} \backslash S^{1}\right\} \cup\left\{P_{\lambda^{f^{\prime}}}\right\},\left\{P_{\lambda^{a}}\right\},\left\{S^{1} \backslash \mu_{\infty}\right\} .
$$

Proof. Follows directly from $N_{G}(H) / H$ action on a character $\chi$ of $H$ above (equations 5.2).

Lemma 5.4. If $H_{1} \in \Sigma_{2,0}$, then the following subgroups $H_{2} \in \Sigma_{2,0}$ are equivalent to $H_{1}$ :

$H_{2}=\phi\left(\left(a, b-a \tilde{d}, e, f, b^{\prime}, e^{\prime}+f^{\prime} \tilde{d}\right)\right)$ for an arbitrary integer $\tilde{d}$.

Fibers $Z_{2,0 ; H_{1}}, Z_{2,0 ; H_{2}}$ over subgroups $H_{1}, H_{2}$ can be canonically identified.

Proof. Since $H_{1}=H_{1}^{*}$, by Proposition 2.16 we need to study only such subgroups $H_{2}$ that there exists an element $g \in G$ such that $\left(H_{2}\right)^{g}=H_{1}$ and $\left.\chi_{1}\right|_{H_{2}^{g} \cap H_{1}}=\left.\chi_{2}^{g}\right|_{H_{2}^{g} \cap H_{1}}$. If $H_{1} \in \Sigma_{2,0}$, then the quotient $G / N_{G}\left(H_{1}\right)$ is generated by

$$
g=\left(\begin{array}{llll}
1 & 0 & 0 & 0 \\
0 & 1 & 1 & 0 \\
0 & 0 & 1 & 0 \\
0 & 0 & 0 & 1
\end{array}\right)
$$

Then it is easy to observe that conjugated subgroups are $\phi\left(\left(a, b-a \tilde{d}, e, f^{\prime}, b^{\prime}, e^{\prime}+f^{\prime} \tilde{d}\right)\right)$. If we denote non-central generators of $H_{2}$ by $\tilde{h}_{1}, \tilde{h}_{2}$, then the characters of the subgroups are related as follows: $\chi_{2}\left(\tilde{h}_{1}\right)=\chi_{1}^{\tilde{d}}\left(h_{1}\right), \quad \chi_{2}\left(\tilde{h}_{2}\right)=\chi_{1}^{\tilde{d}}\left(h_{2}\right)$ and $\chi_{2}(C)=\chi_{1}(C)$. It gives a canonical identification of fibers $Z_{2,0 ; H_{1}}, Z_{2,0 ; H_{2}}$ over subgroups $H_{1}$ and $\mathrm{H}_{2}$. 
6. The CASE OF $\operatorname{rk}_{1}(H)=2, \operatorname{rk}_{2}(H)=1$.

Let us define sets $S_{1}, S_{2}$.

$S_{1}=\left\{\left(a, e, d^{\prime}, e^{\prime}\right) \in \mathbb{Z}^{4} \mid a \neq 0, d \neq 0\right.$ and $\left.|\operatorname{GCD}(a, e)|=1,\left|\operatorname{GCD}\left(d^{\prime}, e^{\prime}\right)\right|=1\right\}$.

$S_{2}=\left\{\left(a, e, d^{\prime}, e^{\prime}\right) \in \mathbb{Z}^{4} \mid a \neq 0, d \neq 0\right.$ and $\left.|\operatorname{GCD}(a, e)|=k_{1},\left|\operatorname{GCD}\left(d^{\prime}, e^{\prime}\right)\right|=k_{2},\left|k_{1} k_{2}\right|>1\right\}$.

Lemma 6.1. There is a canonical bijection $\phi$ from $S_{1} \cup S_{2}$ to $\Sigma_{2,1}$. It maps a tuple $\left(a, e, d^{\prime}, e^{\prime}\right)$ to a subgroup $H$, generated by the following matrices

$$
h_{1}=\left(\begin{array}{cccc}
1 & a & 0 & 0 \\
0 & 1 & 0 & e \\
0 & 0 & 1 & 0 \\
0 & 0 & 0 & 1
\end{array}\right), \quad h_{2}=\left(\begin{array}{cccc}
1 & 0 & 0 & 0 \\
0 & 1 & d^{\prime} & e^{\prime} \\
0 & 0 & 1 & 0 \\
0 & 0 & 0 & 1
\end{array}\right), \quad C=\left(\begin{array}{cccc}
1 & 0 & 0 & 1 \\
0 & 1 & 0 & 0 \\
0 & 0 & 1 & 0 \\
0 & 0 & 0 & 1
\end{array}\right) \text {. }
$$

If $H \in \phi\left(S_{1}\right)$, then we can extend the bijection $\phi$ to the map from

$$
\left(\mathbb{C}^{*}\right)^{2} \times \mu_{N} \times\left\{\mathbb{C}^{*} \backslash \mu_{\infty}\right\}=\left\{(t, r, z, \lambda) \mid z^{a d^{\prime}} \lambda^{a e^{\prime}}=1 \text { and } \lambda \notin \mu_{\infty}\right\}
$$

to $Y_{2,1 ; H}$, which is defined as follows:

$$
t=\chi\left(h_{1}\right), \quad r=\chi\left(h_{2}\right), \quad z=\chi\left(h_{3}\right), \quad \lambda=\chi(C) .
$$

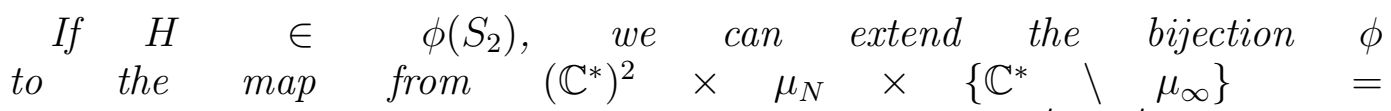
$\left\{(t, r, z, \lambda) \mid z^{a d^{\prime}} \lambda^{a e^{\prime}}=1\right.$, the minimal natural $m$ that $z^{\frac{a d^{\prime} m}{k_{1} k_{2}}} \lambda^{\frac{a e^{\prime} m}{k_{1} k_{2}}}=1$ equals $\left|k_{1} k_{2}\right|$, and $\left.\lambda \notin \mu_{\infty}\right\}$ to $Y_{2,1 ; H}$, which is defined as above.

Proof. The proof goes as follows. First, we prove that if $H \in \Sigma_{2,1}$, then its generators may be chosen in the form given in Lemma 6.1. Then we study characters $\chi_{1}, \chi_{2}: H \rightarrow \mathbb{C}^{*}$ which correspond to equivalent irreducible weight pairs $\left(H, \chi_{1}\right)$ and $\left(H, \chi_{2}\right)$. After that, we obtain conditions for a character $\chi$ that $(H, \chi)$ is an irreducible weight pair.

Let us denote the two generators of $H / H \cap[G, G]$ by $h_{1}, h_{2}$. If $\chi(C)$ is a root of unity, then we need to extend the weight pair $(H, \chi)$ to the pair $\left(H^{\prime}, \chi^{\prime}\right)$ with the $\operatorname{rank}_{2} \operatorname{rk}_{2}\left(H^{\prime}\right)=2$. Hence $\chi(C)$ is not a root of unity. Let us denote by $h_{3}$ the generator of $H \cap[G, G] / Z(G)$. Let us first consider generators $h_{1}$ and $h_{2}$ in the following (general) form:

$$
h_{1}=\left(\begin{array}{llll}
1 & a & b & 0 \\
0 & 1 & d & e \\
0 & 0 & 1 & f \\
0 & 0 & 0 & 1
\end{array}\right), \quad h_{2}=\left(\begin{array}{cccc}
1 & a^{\prime} & b^{\prime} & 0 \\
0 & 1 & d^{\prime} & e^{\prime} \\
0 & 0 & 1 & f^{\prime} \\
0 & 0 & 0 & 1
\end{array}\right)
$$

If $d=d^{\prime}=0$, then the commutator $\left[h_{1}, h_{2}\right]$ is in the center of the group $G$. But then $\left[h_{1}, h_{3}\right]$ and $\left[h_{2}, h_{3}\right]$ can not both be unity, hence $\chi(C)$ is a root 
of unity, which contradicts the earlier statement. Then either $d$ or $d^{\prime}$ is not zero. Hence, the commutator $\left[h_{1}, h_{2}\right]=h_{3}^{k} C^{n}$ for some integers $k, n$. Then $\chi\left(h_{3}\right)^{a d} \chi(C)^{a e^{\prime}}=1$ (in particular, if $e^{\prime}=0$ we obtain that $\chi\left(h_{3}\right)$ is a root of unity). Besides, $\left[h_{1}, h_{3}\right]=\left[h_{2}, h_{3}\right]=1$, otherwise $\left[h_{1}, h_{3}\right]$ and $\left[h_{2}, h_{3}\right]$ are in the center of the group $G$, and $\chi(C)$ is a root of unity. Then the generator $h_{3}$ has to be proportional to $\left[h_{1}, h_{2}\right]$, that is, to the element

$$
\left(\begin{array}{cccc}
1 & 0 & -a & 0 \\
0 & 1 & 0 & f \\
0 & 0 & 1 & 0 \\
0 & 0 & 0 & 1
\end{array}\right)
$$

From $\left[h_{1}, h_{3}\right]=\left[h_{2}, h_{3}\right]=1$ we obtain that either $f=f^{\prime}=0$ or $a=a^{\prime}=0$. Let us consider the case of $f=f^{\prime}=0$ (the other one is treated similarly, if we put integer parameters $\left.f=a, b=e, b^{\prime}=e^{\prime}\right)$. Since $\left[h_{1}, h_{2}\right]=h_{3}^{k} C^{n}$, the generator $h_{3}$ has the following form:

$$
h_{3}=\left(\begin{array}{cccc}
1 & 0 & b^{\prime \prime} & 0 \\
0 & 1 & 0 & 0 \\
0 & 0 & 1 & 0 \\
0 & 0 & 0 & 1
\end{array}\right)
$$

But the element

$$
\left(\begin{array}{llll}
1 & 0 & 1 & 0 \\
0 & 1 & 0 & 0 \\
0 & 0 & 1 & 0 \\
0 & 0 & 0 & 1
\end{array}\right)
$$

belongs to $C_{G}(H)$. Then by Remark 2.6, it coincides with $h_{3}$.

So we can have generators $h_{1}$ and $h_{2}$ in the following form (dividing $h_{1}$ and $h_{2}$ by $h_{3}^{b}$ and $h_{3}^{b^{\prime}}$ correspondingly):

$$
h_{1}=\left(\begin{array}{cccc}
1 & a & 0 & 0 \\
0 & 1 & 0 & e \\
0 & 0 & 1 & 0 \\
0 & 0 & 0 & 1
\end{array}\right), \quad h_{2}=\left(\begin{array}{cccc}
1 & 0 & 0 & 0 \\
0 & 1 & d^{\prime} & e^{\prime} \\
0 & 0 & 1 & 0 \\
0 & 0 & 0 & 1
\end{array}\right)
$$

A subgroup $H$ in this case is not necessarily isolated. Namely, it is not isolated if $|\operatorname{GCD}(a, e)|>1$ or $\left|\operatorname{GCD}\left(d, e^{\prime}\right)\right|>1$.

Now for each $H \in \Sigma_{2,1}$ we describe the fiber $Y_{2,1 ; H}$.

For all subgroups $H \in \Sigma_{2,1}$, the quotient $N_{G}(H) / H$ is generated by 


$$
g=\left(\begin{array}{llll}
1 & 0 & 0 & 0 \\
0 & 1 & 0 & 1 \\
0 & 0 & 1 & 0 \\
0 & 0 & 0 & 1
\end{array}\right)
$$

The action of $g$ on a character $\chi$ is as follows:

$$
\chi^{g}\left(h_{1}\right)=\chi\left(h_{1}\right) \lambda^{-a}, \quad \chi^{g}\left(h_{2}\right)=\chi\left(h_{2}\right), \quad \chi^{g}\left(h_{3}\right)=\chi\left(h_{3}\right) .
$$

If $|\operatorname{GCD}(a, e)|=k_{1},\left|\operatorname{GCD}\left(d, e^{\prime}\right)\right|=k_{2}, \quad\left|k_{1} k_{2}\right|>1$, and $\chi\left(h_{3}\right)^{\frac{a d m}{k_{1} k_{2}}} \chi(C)^{\frac{a e^{\prime} m}{k_{1} k_{2}}}=1$ for an integer $m$, then the elements $h_{2}^{\frac{m_{1}}{k_{1}}}$ and $h_{2}^{\frac{m_{2}}{k_{2}}}$ belong to $C_{G}(H) \backslash H$. It contradicts irreducibility of corresponding induced representation.

Thus, we can see that given the conditions for a character $\chi$ of $H$ formulated in Lemma 6.1, the action above 6.2 is free. Then corresponding representations are irreducible.

Let us denote by $Z_{2,1 ; H}^{(2,1)}=\left\{\left\{(t, \lambda) \mid t \in \mathbb{C}^{*}, \lambda \notin \mu_{\infty}\right\} / \sim\right\}$, by $Z_{2,1 ; H}^{(1,1)}=\{r \in$ $\left.\mathbb{C}^{*}\right\}$, by $Z_{2,1 ; H}^{(2,2)}=\left\{\left\{(z, \lambda) \mid z \in \mathbb{C}^{*}, \lambda \notin \mu_{\infty}\right\} / \sim\right\}$ and by $Z_{2,1 ; H}^{(1,2)}=\left\{\lambda \notin \mu_{\infty}\right\}$, where $\sim$ is defined in 2.12 .

Corollary 6.3. If $H \in \Sigma_{2,1}$, then the fiber $Z_{2,1 ; H}$ of $Z_{2,1}$ over a subgroup $H$ has iterated structure of a bundle, namely:

$$
Z_{2,1 ; H}^{(2,1)} \rightarrow Z_{2,1 ; H}^{(1,2)}, Z_{2,1 ; H}^{(1,1)}, Z_{2,1 ; H}^{(2,2)} \rightarrow Z_{2,1 ; H}^{(1,2)}
$$

We describe fibers of these bundles consecutively in coordinates $(t, r, z, \lambda)$ :

$$
\left\{E_{\lambda^{a}}\right\}, \mathbb{C}^{*}, \mu_{a d^{\prime}},\left\{\mathbb{C}^{*} \backslash S^{1}\right\} \cup\left\{P_{\lambda^{a}}\right\}, \mathbb{C}^{*}, \mu_{a d^{\prime}},\left\{S^{1} \backslash \mu_{\infty}\right\} .
$$

Proof. Follows directly from $N_{G}(H) / H$ action on a character $\chi$ of a subgroup $H$ above (equations 6.2).

We obtain the value of $z=\left(\lambda^{-a e^{\prime}}\right)^{\frac{1}{a d^{\prime}}}$ from $z^{a d^{\prime}} \lambda^{a e^{\prime}}=1$.

Lemma 6.4. If $H_{1} \in \Sigma_{2,1}$, then the following subgroups are equivalent to $H_{1}$ : $H_{2}=\phi\left(\left(a, e, d^{\prime}, e^{\prime}-d^{\prime} \tilde{f}\right)\right)$ for an arbitrary integer $\tilde{f}$.

If $H_{1} \in \phi\left(S_{2}\right)$, then there is also a finite set of subgroups which are $F$ equivalent to $H_{1}$.

Fibers $Z_{2,1 ; H_{1}}$ and $Z_{2,1 ; H_{2}}$ over equivalent subgroups may be identified canonically. 
Proof. First, by Proposition 2.16](i)] we consider $G / N_{G}\left(H_{1}^{*}\right)$ action on $H_{1}$. The quotient $G / N_{G}\left(H_{1}^{*}\right)$ is generated by

$$
g=\left(\begin{array}{llll}
1 & 0 & 0 & 0 \\
0 & 1 & 0 & 0 \\
0 & 0 & 1 & 1 \\
0 & 0 & 0 & 1
\end{array}\right)
$$

It is easy to compute that conjugated subgroups are $\phi\left(\left(a, e, d^{\prime}, e^{\prime}-d^{\prime} \tilde{f}\right)\right)$. Let us denote generators of $H_{2}$ which generate $H_{2} /\left(H_{2} \cap[G, G]\right)$ by $\tilde{h}_{1}, \tilde{h}_{2}$, and we denote by $\tilde{h}_{3}$ the element which generates $\left(H_{2} \cap[G, G]\right) / Z(G)$. Then characters of the subgroups are related as follows: $\chi_{2}\left(\tilde{h}_{1}\right)=\chi_{1}^{\tilde{f}}\left(h_{1}\right), \chi_{2}\left(\tilde{h}_{2}\right)=$ $\chi_{1}^{\tilde{f}}\left(h_{2}\right), \chi_{2}\left(\tilde{h}_{3}\right)=\chi_{1}^{\tilde{f}}\left(h_{3}\right)$, and $\chi_{2}(C)=\chi_{1}(C)$. It gives a canonical identification of fibers $Z_{2,1 ; H_{1}}$ and $Z_{2,1 ; H_{2}}$ over subgroups $H_{1}$ and $H_{2}$.

If $H_{1} \in \phi\left(S_{2}\right)$, then $H_{1}$ is not isolated. By Proposition [2.16(ii)] we need to consider also equivalent irreducible weight pairs $\left(H_{1}, \chi_{1}\right)$ and $\left(H_{2}, \chi_{2}\right)$ such that $H_{1}^{*}=H_{2}^{*}$ and $H_{1} \neq H_{2}$. Clearly, we can only possibly extract roots from generators $h_{1}$ and $h_{2}$. The condition $z^{a d^{\prime}} \lambda^{a e^{\prime}}=1$ (which here stands for $\left.\chi_{1}\left(\left[h_{1}, h_{2}\right]\right)=1\right)$ holds as well for $H_{2}$, since $h_{3}=\tilde{h}_{3}, \chi_{1}\left(h_{3}\right)=\chi_{2}\left(h_{3}\right)=z$ and $\chi_{1}(C)=\chi_{2}(C)=\lambda$. Let us denote $\operatorname{GCD}(a, e)=k_{1}$ and $\operatorname{GCD}\left(d^{\prime}, e^{\prime}\right)=k_{2}$. Then for every divisor $m_{1}$ of $k_{1}$, a subgroup $\phi\left(\left(\frac{a}{m_{1}}, \frac{e}{m_{1}}, d^{\prime} m_{1}, e^{\prime} m_{1}\right)\right)$ with a character $\chi_{2}$ defined by $\chi_{1}\left(h_{1}\right)=\chi_{2}\left(\tilde{h}_{1}\right)^{m_{1}}, \chi_{1}\left(h_{2}\right)^{m_{1}}=\chi\left(\tilde{h}_{2}\right)$ is $F$-equivalent to $H_{1}$. Similarly, a subgroup $\phi\left(\left(a m_{2}, e m_{2}, \frac{d^{\prime}}{m_{2}}, \frac{e^{\prime}}{m_{2}}\right)\right)$ with a character $\chi_{2}$ defined by $\chi_{1}\left(h_{1}\right)^{m_{2}}=\chi\left(\tilde{h}_{1}\right), \chi_{1}\left(h_{2}\right)=\chi\left(\tilde{h}_{2}\right)^{m_{2}}$ is $F$-equivalent to $H_{1}$.

7. The CASE OF $\operatorname{rk}_{1}(H)=1, \operatorname{rk}_{2}(H)=2$.

Let us define sets $S_{1}, S_{2}, S_{3}$, and $A$.

$$
\begin{gathered}
S_{1}=\left\{\left(a, d, f, b, e, b^{\prime}, e^{\prime}\right) \in \mathbb{Z}^{7}\left|a \neq 0, f \neq 0, e^{\prime} \neq 0, b^{\prime} \neq 0,\right| b|<| b^{\prime}|,| e|<| e^{\prime} \mid\right\}, \\
S_{2}=\left\{\left(a, d, f, b, e, b^{\prime}, e^{\prime}\right) \in \mathbb{Z}^{7}\left|a \neq 0, d \neq 0, f=0, e^{\prime} \neq 0, b^{\prime}=1, b=0,\right| e|<| e^{\prime} \mid\right\}, \\
S_{3}=\left\{\left(a, d, f, b, e, b^{\prime}, e^{\prime}\right) \in \mathbb{Z}^{7}\left|a=0, d \neq 0, f \neq 0, b^{\prime} \neq 0, e^{\prime}=1,\right| b|<| b^{\prime} \mid, e=0\right\}, \\
A=\left\{\left(a, d, f, b, e, b^{\prime}, e^{\prime}\right) \in \mathbb{Z}^{7} \mid a=f=0, d=1, b=0, e=0, b^{\prime}=1, e^{\prime}=1\right\} .
\end{gathered}
$$$$
\text { Let } N=\operatorname{GCD}\left(\left|a e^{\prime}\right|,\left|f b^{\prime}\right|\right) \text {. }
$$ 
Lemma 7.1. There is a canonical bijection $\phi$ from $S_{1} \cup S_{2} \cup S_{3} \cup A$ to $\Sigma_{1,2}$. It maps a tuple $\left(a, d, f, b, e, b^{\prime}, e^{\prime}\right)$ to a subgroup $H$, generated by the following matrices

$$
h_{1}=\left(\begin{array}{llll}
1 & a & b & 0 \\
0 & 1 & d & e \\
0 & 0 & 1 & f \\
0 & 0 & 0 & 1
\end{array}\right), \quad h_{2}=\left(\begin{array}{cccc}
1 & 0 & b^{\prime} & 0 \\
0 & 1 & 0 & 0 \\
0 & 0 & 1 & 0 \\
0 & 0 & 0 & 1
\end{array}\right), \quad h_{3}=\left(\begin{array}{cccc}
1 & 0 & 0 & 0 \\
0 & 1 & 0 & e^{\prime} \\
0 & 0 & 1 & 0 \\
0 & 0 & 0 & 1
\end{array}\right), \quad C=\left(\begin{array}{cccc}
1 & 0 & 0 & 1 \\
0 & 1 & 0 & 0 \\
0 & 0 & 1 & 0 \\
0 & 0 & 0 & 1
\end{array}\right)
$$

If $H \in \phi\left(S_{1}\right) \cup \phi\left(S_{2}\right) \cup \phi\left(S_{3}\right)$, we can extend the bijection $\phi$ to the map from

$\mathbb{C}^{*} \times\left\{\mathbb{C}^{*} \backslash \mu_{\infty}\right\} \times\left\{\mathbb{C}^{*} \backslash \mu_{\infty}\right\} \times\left\{\mu_{N}\right\}=\left\{(t, z, w, \lambda) \mid z, w \notin \mu_{\infty}, \lambda^{N}=1\right\}$

to $Y_{1,2 ; H}$, which is defined as follows:

$$
t=\chi\left(h_{1}\right), \quad z=\chi\left(h_{2}\right), \quad w=\chi\left(h_{3}\right), \quad \lambda=\chi(C) .
$$

If $H \in \phi(A)$, then $H$ is abelian (and there is only one such a subgroup). We can extend the bijection $\phi$ to the map from

$$
\begin{gathered}
\mathbb{C}^{*} \times\left\{\mathbb{C}^{*} \backslash \mu_{\infty}\right\} \times\left\{\mathbb{C}^{*} \backslash \mu_{\infty}\right\} \times \mathbb{C}^{*}=\left\{(t, z, w, \lambda) \mid z, w \notin \mu_{\infty}\right\} \cup \\
\mathbb{C}^{*} \times \mathbb{C}^{*} \times \mathbb{C}^{*} \times\left\{\mathbb{C}^{*} \backslash \mu_{\infty}\right\}=\left\{(t, z, w, \lambda) \mid \lambda \notin \mu_{\infty}\right\}
\end{gathered}
$$

to $Y_{1,2 ; H}$, which is defined as above.

Proof. The proof goes as follows. We consider cases of non-abelian subgroups and the abelian subgroup separately. We study characters $\chi_{1}, \chi_{2}: H \rightarrow \mathbb{C}^{*}$ which correspond to equivalent irreducible weight pairs $\left(H, \chi_{1}\right)$ and $\left(H, \chi_{2}\right)$. Then we obtain conditions for a character $\chi$ that $(H, \chi)$ is an irreducible weight pair, and we compute the fiber $Y_{1,2 ; H}$ over a subgroup $H$.

Clearly, a subgroup $H$ with $\operatorname{ranks} \operatorname{rk}_{1}(H)=1, \operatorname{rk}_{1}(H)=2$ can be generated as follows:

$$
h_{1}=\left(\begin{array}{llll}
1 & a & b & 0 \\
0 & 1 & d & e \\
0 & 0 & 1 & f \\
0 & 0 & 0 & 1
\end{array}\right), \quad h_{2}=\left(\begin{array}{cccc}
1 & 0 & b^{\prime} & 0 \\
0 & 1 & 0 & 0 \\
0 & 0 & 1 & 0 \\
0 & 0 & 0 & 1
\end{array}\right), \quad h_{3}=\left(\begin{array}{cccc}
1 & 0 & 0 & 0 \\
0 & 1 & 0 & e^{\prime} \\
0 & 0 & 1 & 0 \\
0 & 0 & 0 & 1
\end{array}\right)
$$

We have $\chi\left(\left[h_{1}, h_{2}\right]\right)=\chi(C)^{f b^{\prime}}=1$ and $\chi\left(\left[h_{1}, h_{3}\right]\right)=\chi(C)^{a e^{\prime}}=1$. From irreducibility criterion $S(H, \chi)=H$ we conclude that $b^{\prime}, e^{\prime}$ have to be minimal natural numbers satisfying the condition $\chi(C)^{f b^{\prime}}=\chi(C)^{a e^{\prime}}=1$ (otherwise we can extend the weight pair). Obviously, integers $b^{\prime}$ and $e^{\prime}$ have to be non-zero and $|b|,|e|$ may be chosen to be smaller than $\left|b^{\prime}\right|,\left|e^{\prime}\right|$. If $f=0$, then $b^{\prime}=0$ from the condition $S(H, \chi)=H$, and hence $b=0$ (similarly for $a=0$ we have $e=0)$.

(i). First, let us consider the case of a subgroup $H \in \Sigma_{1,2} \backslash \phi(A)$. Then either $a \neq 0$, or $f \neq 0$, and hence, $\chi(C)$ is a root of unity. Let us consider the first 
case with $a \neq 0$ (the other one with $f \neq 0$ is treated similarly). If $d=0$ then the weight pair may be extended to the one with $\operatorname{ranks} \operatorname{rk}_{1}(H)=\operatorname{rk}_{2}(H)=2$. Hence, $d$ is non-zero.

In this case, the quotient $N_{G}(H) / H$ is generated by

$$
g_{1}=\left(\begin{array}{cccc}
1 & 0 & 0 & 0 \\
0 & 1 & d^{\prime} & 0 \\
0 & 0 & 1 & 0 \\
0 & 0 & 0 & 1
\end{array}\right), \quad g_{2}=\left(\begin{array}{cccc}
1 & 0 & 0 & 0 \\
0 & 1 & 0 & 0 \\
0 & 0 & 1 & f^{\prime} \\
0 & 0 & 0 & 1
\end{array}\right)
$$

where $d^{\prime}$ in $g_{1}$ is such a minimal natural number that $a d^{\prime}$ is divisible by $b^{\prime}$ and $f d^{\prime}$ is divisible by $e^{\prime}$, and $f^{\prime}$ in $g_{2}$ is a minimal natural number that $f^{\prime} d$ is divisible by $e^{\prime}$.

In this case a subgroup $H$ is not necessarily isolated.

Generators $g_{1}, g_{2}$ act on a character $\chi$ as follows:

$$
\begin{gathered}
\chi^{g_{1}}\left(h_{1}\right)=\chi\left(h_{1}\right) \chi\left(h_{2}\right)^{-\frac{a d^{\prime}}{b^{\prime}}} \chi\left(h_{3}\right)^{\frac{f d^{\prime}}{e^{\prime}}}, \\
\chi^{g_{1}}\left(h_{2}\right)=\chi\left(h_{2}\right), \quad \chi^{g_{1}}\left(h_{3}\right)=\chi\left(h_{3}\right) \\
\text { and } \\
\chi^{g_{2}}\left(h_{1}\right)=\chi\left(h_{1}\right) \chi\left(h_{3}\right)^{-\frac{f^{\prime} d}{e^{\prime}}} \chi(C)^{-b f^{\prime}}, \\
\chi^{g_{2}}\left(h_{2}\right)=\chi\left(h_{2}\right) \chi(C)^{-b^{\prime} f^{\prime}}, \quad \chi^{g_{2}}\left(h_{3}\right)=\chi\left(h_{3}\right) .
\end{gathered}
$$

From irreducibility criterion $S(H, \chi)=H$ we obtain that neither $\chi\left(h_{2}\right)$ nor $\chi\left(h_{3}\right)$ is a root of unity, otherwise we can extend the weight pair $(H, \chi)$ to the weight pair $\left(H^{\prime}, \chi^{\prime}\right)$ with $\operatorname{rk}_{1}\left(H^{\prime}\right)=\operatorname{rk}_{2}\left(H^{\prime}\right)=2$. If these conditions are satisfied, then the action above is free, and corresponding representations are irreducible.

(ii). Now let us consider the case of the abelian subgroup $H \in \phi(A)$ (all the other cases were proven to correspond to non-abelian subgroups). Since $\left[h_{1}, h_{2}\right]=\left[h_{1}, h_{3}\right]=1$, we have $a=f=0$ for a generator $h_{1}$ in the following form:

$$
\left(\begin{array}{llll}
1 & a & b & 0 \\
0 & 1 & d & e \\
0 & 0 & 1 & f \\
0 & 0 & 0 & 1
\end{array}\right)
$$

From Lemma 2.10 it follows that since $H$ is abelian, it is isolated. Hence, generators of $H$ have the following form: 


$$
h_{1}=\left(\begin{array}{llll}
1 & 0 & 0 & 0 \\
0 & 1 & 1 & 0 \\
0 & 0 & 1 & 0 \\
0 & 0 & 0 & 1
\end{array}\right), \quad h_{2}=\left(\begin{array}{llll}
1 & 0 & 1 & 0 \\
0 & 1 & 0 & 0 \\
0 & 0 & 1 & 0 \\
0 & 0 & 0 & 1
\end{array}\right), \quad h_{3}=\left(\begin{array}{llll}
1 & 0 & 0 & 0 \\
0 & 1 & 0 & 1 \\
0 & 0 & 1 & 0 \\
0 & 0 & 0 & 1
\end{array}\right)
$$

Since $N_{G}(H)=G$, the quotient $N_{G}(H) / H$ is generated by

$$
g_{1}=\left(\begin{array}{llll}
1 & 1 & 0 & 0 \\
0 & 1 & 0 & 0 \\
0 & 0 & 1 & 0 \\
0 & 0 & 0 & 1
\end{array}\right), \quad g_{2}=\left(\begin{array}{cccc}
1 & 0 & 0 & 0 \\
0 & 1 & 0 & 0 \\
0 & 0 & 1 & 1 \\
0 & 0 & 0 & 1
\end{array}\right)
$$

Then $g_{1}$ and $g_{2}$ act on a character $\chi$ as follows:

$$
\begin{gathered}
\chi^{g_{1}}\left(h_{1}\right)=\chi\left(h_{1}\right) \chi\left(h_{2}\right), \quad \chi^{g_{1}}\left(h_{2}\right)=\chi\left(h_{2}\right), \quad \chi^{g_{1}}\left(h_{3}\right)=\chi\left(h_{3}\right) \lambda \\
\text { and } \\
\chi^{g_{2}}\left(h_{1}\right)=\chi\left(h_{1}\right) \chi\left(h_{3}\right)^{-1}, \quad \chi^{g_{2}}\left(h_{2}\right)=\chi\left(h_{2}\right) \lambda^{-1}, \quad \chi^{g_{2}}\left(h_{3}\right)=\chi\left(h_{3}\right) .
\end{gathered}
$$

Then from irreducibility criterion $S(H, \chi)=H$ we obtain that $\chi\left(h_{2}\right), \chi\left(h_{3}\right)$ or $\chi(C)$ is not a root of unity. If these conditions are satisfied, then the action above is free, and corresponding representations are irreducible.

Let us denote by $Z_{1,2 ; H}^{(2)}=\left\{\left\{(t, z, w, \lambda) \mid t \in \mathbb{C}^{*}, z, w \in\left\{\mathbb{C}^{*} \backslash \mu_{\infty}\right\}^{2}, \lambda^{N}=\right.\right.$ $1\} / \sim\}$, by $Z_{1,2 ; H}^{(1)}=\left\{(z, w, \lambda) \mid z, w \in\left\{\mathbb{C}^{*} \backslash \mu_{\infty}\right\}^{2}, \lambda^{N}=1\right\}$, where $\sim$ is defined in 2.12 .

Let us denote by $Z_{1,2 \text {, ab; } H}^{(2)}=\left\{\left\{(t, z, w, \lambda) \mid t \in \mathbb{C}^{*}\right.\right.$, z or $w$ or $\left.\left.\lambda \notin \mu_{\infty}\right\} / \sim\right\}$, by $Z_{1,2, \mathrm{ab} ; H}^{(1)}=\left\{(z, w, \lambda) \mid z\right.$ or $w$ or $\left.\lambda \notin \mu_{\infty}\right\}$, where $\sim$ is defined in 2.12.

Corollary 7.2. If $H \in \Sigma_{1,2} \backslash \phi(A)$, then the fiber $Z_{1,2 ; H}$ of $Z_{1,2}$ over a subgroup $H$ has the following iterated structure of a bundle, namely:

$$
Z_{1,2 ; H}^{(2)} \rightarrow Z_{1,2 ; H}^{(1)}
$$

We describe fibers of this bundle consecutively in coordinates $(t, z, w, \lambda)$.

$$
T_{z^{\frac{a d^{\prime}}{b^{\prime}}} w^{\frac{f d^{\prime}}{e^{\prime}}}, w^{\frac{f^{\prime} d}{e^{\prime}}} \lambda^{b f^{\prime}}},\left\{\mathbb{C}^{*} \backslash \mu_{\infty}\right\},\left\{\mathbb{C}^{*} \backslash \mu_{\infty}\right\},\left\{\mu_{N}\right\}
$$

If $H \in \phi(A)$, then the fiber $Z_{1,2 ; H}$ of $Z_{1,2}$ over a subgroup $H$ has the following iterated structure of a bundle, namely:

$$
Z_{1,2 a b ; H}^{(2)} \underset{25}{\rightarrow} Z_{1,2 a b ; H}^{(1)}
$$


We describe fibers of this bundle consecutively in coordinates $(t, z, w, \lambda)$.

$$
\begin{aligned}
T_{z, w},\left\{E_{\lambda} \backslash \mu_{\infty}\right\},\left\{E_{\lambda} \backslash \mu_{\infty}\right\},\left\{\mathbb{C}^{*} \backslash S^{1}\right\} \cup \\
T_{z, w},\left\{P_{\lambda} \backslash \mu_{\infty}\right\},\left\{P_{\lambda} \backslash \mu_{\infty}\right\},\left\{S^{1} \backslash \mu_{\infty}\right\} \cup \\
\left\{E_{z}\right\},\left\{E_{\lambda} \backslash \mu_{\infty}\right\},\left\{E_{\lambda} \cap \mu_{\infty}\right\},\left\{\mathbb{C}^{*} \backslash S^{1}\right\} \cup \\
\left\{E_{z}\right\},\left\{P_{\lambda} \backslash \mu_{\infty}\right\},\left\{P_{\lambda} \cap \mu_{\infty}\right\},\left\{S^{1} \backslash \mu_{\infty}\right\} \cup \\
\left\{E_{w}\right\},\left\{E_{\lambda} \cap \mu_{\infty}\right\},\left\{E_{\lambda} \backslash \mu_{\infty}\right\},\left\{\mathbb{C}^{*} \backslash S^{1}\right\} \cup \\
\left\{E_{w}\right\},\left\{P_{\lambda} \cap \mu_{\infty}\right\},\left\{P_{\lambda} \backslash \mu_{\infty}\right\},\left\{S^{1} \backslash \mu_{\infty}\right\} \cup \\
\left\{\mathbb{C}^{*}\right\},\left\{E_{\lambda} \cap \mu_{\infty}\right\},\left\{E_{\lambda} \cap \mu_{\infty}\right\},\left\{\mathbb{C}^{*} \backslash S^{1}\right\} \cup \\
\left\{\mathbb{C}^{*}\right\},\left\{P_{\lambda} \cap \mu_{\infty}\right\},\left\{P_{\lambda} \cap \mu_{\infty}\right\},\left\{S^{1} \backslash \mu_{\infty}\right\} \cup \\
\quad\left\{T_{z, w}\right\},\left\{\mathbb{C}^{*} \backslash \mu_{\infty}\right\},\left\{\mathbb{C}^{*} \backslash \mu_{\infty}\right\},\left\{\mu_{\infty}\right\} \cup \\
\left\{T_{z, w}\right\},\left\{\mathbb{C}^{*} \backslash \mu_{\infty}\right\},\left\{\mathbb{C}^{*} \backslash \mu_{\infty}\right\},\left\{\mu_{\infty}\right\} .
\end{aligned}
$$

Proof. Follows directly from $N_{G}(H) / H$ action on a character $\chi$ of $H$ above.

Lemma 7.3. If $H \in \Sigma_{1,2} \backslash \phi(A)$, then there are only subgroups, which are $F$-equivalent to $H$ (finitely many subgroups).

If $H \in \phi(A)$, then the corresponding subgroup is abelian and normal, and it is not equivalent to any other subgroups.

Fibers $Z_{1,2 ; H_{1}}$ and $Z_{1,2 ; H_{2}}$ over equivalent subgroups $H_{1}$ and $H_{2}$ may be identified canonically.

Proof. Let us consider $H_{1} \in \Sigma_{1,2} \backslash \phi(A)$.

Since $N_{G}\left(H_{1}^{*}\right)=G$, by Proposition [2.16 (ii)] we consider only equivalent irreducible weight pairs $\left(H_{1}, \chi_{1}\right)$ and $\left(H_{2}, \chi_{2}\right)$ with $H_{1}^{*}=H_{2}^{*}$ and $H_{1} \neq H_{2}$.

We can only possibly extract roots from generators $h_{1}, h_{2}$, and $h_{3}$. Then if there is an element $g_{1} \in G \backslash H_{1}$ such that $g_{1}^{k_{1}}=h_{1}$, then by Proposition 2.16](ii)] for any divisor $m_{1}$ of $k_{1}$ there exists a finite set of exponents of generators $h_{2}$ and $h_{3}$ such that $S\left(H_{2}, \chi_{2}\right)=H_{2}$. Since $g_{2}^{b^{\prime}}=h_{2}$ and $g_{3}^{e^{\prime}}=h_{3}$, where

$$
g_{2}=\left(\begin{array}{llll}
1 & 0 & 1 & 0 \\
0 & 1 & 0 & 0 \\
0 & 0 & 1 & 0 \\
0 & 0 & 0 & 1
\end{array}\right), \quad g_{3}=\left(\begin{array}{cccc}
1 & 0 & 0 & 0 \\
0 & 1 & 0 & 1 \\
0 & 0 & 1 & 0 \\
0 & 0 & 0 & 1
\end{array}\right) \text {, }
$$

then for any divisor $m_{2}$ of $b^{\prime}$ and any divisor $m_{3}$ of $e^{\prime}$, there also exists a finite set of corresponding exponents. We omit concrete expressions of parameters of equivalent weight pairs for their cumbersomeness (but they are easy to compute). 
Since $a, d, f, b^{\prime}, e^{\prime}$ are fixed, once we fixed the exponents of generators of $H_{1}$, there are only parameters $b<\left|b^{\prime}\right|$ and $e<\left|e^{\prime}\right|$, which yet can produce equivalent irreducible weight pairs. Let us consider $H_{2}=\phi\left(\left(a, d, f, 0,0, b^{\prime}, e^{\prime}\right)\right)$ with a character $\chi_{2}$ defined by $\chi_{2}\left(\tilde{h}_{1}\right)=\chi_{1}\left(h_{1}\right) \chi_{1}\left(h_{2}\right)^{-\frac{b}{b^{\prime}}} \chi_{1}\left(h_{3}\right)^{-\frac{e}{e^{\prime}}}$ and $\chi_{1}\left(\left\langle h_{2}, h_{3}, C\right\rangle\right)=\chi_{2}\left(\left\langle h_{2}, h_{3}, C\right\rangle\right)$. Then $H_{1}^{*}=H_{2}^{*}$ and $\left.\chi_{1}\right|_{H_{1} \cap H_{2}}=\left.\chi_{2}\right|_{H_{1} \cap H_{2}}$. All such irreducible weight pairs are $F$-equivalent.

If $H \in \phi(A)$, then the corresponding subgroup $H$ is normal and abelian. Hence, $H$ is isolated, and by Proposition 2.16 the weight pair $(H, \chi)$ can not be equivalent to any irreducible weight pair with a different subgroup $\mathrm{H}_{2}$.

\section{The CASE OF $\operatorname{rk}_{1}(H)=2, \operatorname{rk}_{2}(H)=2$.}

Let us define sets $S_{1}, S_{2}, S_{3}$ and $S_{4}$.

$$
\begin{gathered}
S_{1}=\left\{\left(a, f, b, e, d^{\prime}, f^{\prime}, b^{\prime}, e^{\prime}, b^{\prime \prime}, e^{\prime \prime}\right) \in \mathbb{Z}^{10} \mid a \neq 0, d \neq 0, f^{\prime} \neq 0, f \neq 0, b^{\prime \prime} \neq 0, e^{\prime \prime} \neq 0,\right. \\
\left.d^{\prime} f \vdots e^{\prime \prime}, d^{\prime} a \vdots b^{\prime \prime},|b|<\left|b^{\prime \prime}\right|,|e|<\left|e^{\prime \prime}\right|,\left|b^{\prime}\right|<\left|b^{\prime \prime}\right|,\left|e^{\prime}\right|<\left|e^{\prime \prime}\right|\right\}, \\
S_{2}=\left\{\left(a, f, b, e, d^{\prime}, f^{\prime}, b^{\prime}, e^{\prime}, b^{\prime \prime}, e^{\prime \prime}\right) \in \mathbb{Z}^{10} \mid a \neq 0, d \neq 0, f=f^{\prime}=0, e^{\prime \prime} \neq 0, b^{\prime \prime}=1,\right. \\
\left.b=b^{\prime}=0,|e|<\left|e^{\prime \prime}\right|,\left|e^{\prime}\right|<\left|e^{\prime \prime}\right|\right\}, \\
S_{3}=\left\{\left(a, f, b, e, d^{\prime}, f^{\prime}, b^{\prime}, e^{\prime}, b^{\prime \prime}, e^{\prime \prime}\right) \in \mathbb{Z}^{10} \mid a=0, d \neq 0, f^{\prime} \neq 0, f=0, e^{\prime \prime}=1, b^{\prime \prime} \neq 0,\right. \\
\left.e=e^{\prime}=0,\left|b^{\prime}\right|<\left|b^{\prime \prime}\right|,|b|<\left|b^{\prime \prime}\right|\right\}, \\
S_{4}=\left\{\left(a, f, b, e, d^{\prime}, f^{\prime}, b^{\prime}, e^{\prime}, b^{\prime \prime}, e^{\prime \prime}\right) \in \mathbb{Z}^{10} \mid a \neq 0, f^{\prime} \neq 0, d^{\prime}=0, b^{\prime \prime} \neq 0,\right. \\
\left.e^{\prime \prime} \neq 0,|b|<\left|b^{\prime \prime}\right|,|e|<\left|e^{\prime \prime}\right|,\left|b^{\prime}\right|<\left|b^{\prime \prime}\right|,\left|e^{\prime}\right|<\left|e^{\prime \prime}\right|\right\} .
\end{gathered}
$$

Let $N=\operatorname{GCD}\left(f b^{\prime \prime}, f^{\prime} b^{\prime \prime}, a e^{\prime \prime}\right)$. Let us denote by $C_{z, w}$ the following curve: $\left\{w^{\frac{-d^{\prime} f}{e^{\prime \prime}}} z^{\frac{-d^{\prime} a}{b^{\prime \prime}}} \lambda^{a e^{\prime}+b f^{\prime}-b^{\prime} f}=1 \mid z, w \in\left(\mathbb{C}^{*}\right)^{2}, z\right.$ or $\left.w \notin \mu_{\infty}, \lambda^{N}=1\right\}$. Let us denote by $C_{z, w, s i n g}$ the following manifold: $\left\{w, z \in C_{z, w}\right.$ such that $z \in$ $\mu_{\infty}$ and $\left.w \in\left\{S^{1} \backslash \mu_{\infty}\right\}\right\}$. Let $N_{2}$ be a minimal natural number such that if $z^{\frac{-d^{\prime} a}{b^{\prime \prime}}} \lambda^{a e^{\prime}}=1$, then $z^{N_{2}}=1$. Let $N_{3}$ be a minimal natural number such that if $w^{\frac{-d^{\prime} f}{e^{\prime \prime}}} \lambda^{b f^{\prime}-b^{\prime} f}=1$, then $w^{N_{3}}=1$.

Lemma 8.1. There is a canonical bijection $\phi$ from $S_{1} \cup S_{2} \cup S_{3} \cup S_{4}$ to $\Sigma_{2,2}$. It maps a tuple $\left(a, f, b, e, d^{\prime}, f^{\prime}, b^{\prime}, e^{\prime}, b^{\prime \prime}, e^{\prime \prime}\right)$ to a subgroup $H$, generated by the following matrices

$$
h_{1}=\left(\begin{array}{cccc}
1 & a & b & 0 \\
0 & 1 & 0 & e \\
0 & 0 & 1 & f \\
0 & 0 & 0 & 1
\end{array}\right), \quad h_{2}=\left(\begin{array}{cccc}
1 & 0 & b^{\prime} & 0 \\
0 & 1 & d^{\prime} & e^{\prime} \\
0 & 0 & 1 & f^{\prime} \\
0 & 0 & 0 & 1
\end{array}\right),
$$




$$
h_{3}=\left(\begin{array}{cccc}
1 & 0 & b^{\prime \prime} & 0 \\
0 & 1 & 0 & 0 \\
0 & 0 & 1 & 0 \\
0 & 0 & 0 & 1
\end{array}\right), \quad h_{4}=\left(\begin{array}{cccc}
1 & 0 & 0 & 0 \\
0 & 1 & 0 & e^{\prime \prime} \\
0 & 0 & 1 & 0 \\
0 & 0 & 0 & 1
\end{array}\right), \quad C=\left(\begin{array}{cccc}
1 & 0 & 0 & 1 \\
0 & 1 & 0 & 0 \\
0 & 0 & 1 & 0 \\
0 & 0 & 0 & 1
\end{array}\right)
$$

If $H \in \phi\left(S_{1}\right)$, we can extend the bijection $\phi$ to the map from

$$
\mathbb{C}^{*} \times \mathbb{C}^{*} \times C_{z, w} \times \mu_{N}=\left\{(t, s, z, w, \lambda) \mid w^{\frac{-d^{\prime} f}{e^{\prime \prime}}} z^{\frac{-d^{\prime} a}{b^{\prime \prime}}} \lambda^{a e^{\prime}+b f^{\prime}-b^{\prime} f}=1, \lambda \in \mu_{N}\right\}
$$

to $Y_{2,2 ; H}$, which is defined as follows:

$$
t=\chi\left(h_{1}\right), \quad s=\chi\left(h_{2}\right), \quad z=\chi\left(h_{3}\right), \quad w=\chi\left(h_{4}\right), \quad \lambda=\chi(C) .
$$

If $H \in \phi\left(S_{2}\right)$, we can extend the bijection $\phi$ to the map from

$$
\mathbb{C}^{*} \times \mathbb{C}^{*} \times \mu_{N_{2}} \times\left\{\mathbb{C}^{*} \backslash \mu_{\infty}\right\} \times \mu_{a e^{\prime \prime}}=\left\{(t, s, z, w, \lambda) \mid z^{\frac{-d^{\prime} a}{b^{\prime \prime}}} \lambda^{a e^{\prime}}=1, \lambda \in \mu_{a e^{\prime \prime}}\right\}
$$

to $Y_{2,2 ; H}$, which is defined as above.

If $H \in \phi\left(S_{3}\right)$, we can extend the bijection $\phi$ to the map from

$$
\mathbb{C}^{*} \times \mathbb{C}^{*} \times\left\{\mathbb{C}^{*} \backslash \mu_{\infty}\right\} \times \mu_{N_{3}} \times \mu_{f^{\prime} b^{\prime \prime}}=\left\{(t, s, z, w, \lambda) \mid w^{\frac{-d^{\prime} f}{e^{\prime \prime}}} \lambda^{b f^{\prime}-b^{\prime} f}=1, \lambda \in \mu_{f^{\prime} b^{\prime \prime}}\right\}
$$

to $Y_{2,2 ; H}$, which is defined as above.

If $H \in \phi\left(S_{4}\right)$, we can extend the bijection $\phi$ to the map from

$$
\mathbb{C}^{*} \times \mathbb{C}^{*} \times\left\{\mathbb{C}^{*} \backslash \mu_{\infty}\right\} \times \mathbb{C}^{*} \times \mu_{N}=\left\{(t, s, z, w, \lambda) \mid z \text { or } w \notin \mu_{\infty}, \lambda \in \mu_{N}\right\}
$$

to $Y_{2,2 ; H}$, which is defined as above.

Proof. The proof goes as follows. We consider separately cases of $H \in\left(\phi\left(S_{1}\right) \cup \phi\left(S_{2}\right) \cup \phi\left(S_{3}\right)\right)$ and the case of $H \in \phi\left(S_{4}\right)$. Then we study characters $\chi_{1}, \chi_{2}: H \rightarrow \mathbb{C}^{*}$ which correspond to equivalent irreducible weight pairs $\left(H, \chi_{1}\right)$ and $\left(H, \chi_{2}\right)$. Then we obtain conditions for a character $\chi$ that $(H, \chi)$ is an irreducible weight pair, and we compute the fiber $Y_{2,2 ; H}$.

(1). If $H \in \phi\left(S_{1}\right), \phi\left(S_{2}\right)$ or $\phi\left(S_{3}\right)$, then all cases of such subgroups are treated similarly, and without loss of generality we consider the case of a subgroup $H \in \phi\left(S_{1}\right)$.

If $H \in \phi\left(S_{1}\right)$, then a subgroup $H$ can be generated as follows:

$$
h_{1}=\left(\begin{array}{cccc}
1 & a & b & 0 \\
0 & 1 & 0 & e \\
0 & 0 & 1 & f \\
0 & 0 & 0 & 1
\end{array}\right), \quad h_{2}=\left(\begin{array}{cccc}
1 & 0 & b^{\prime} & 0 \\
0 & 1 & d^{\prime} & e^{\prime} \\
0 & 0 & 1 & f^{\prime} \\
0 & 0 & 0 & 1
\end{array}\right)
$$




$$
h_{3}=\left(\begin{array}{cccc}
1 & 0 & b^{\prime \prime} & 0 \\
0 & 1 & 0 & 0 \\
0 & 0 & 1 & 0 \\
0 & 0 & 0 & 1
\end{array}\right), \quad h_{4}=\left(\begin{array}{cccc}
1 & 0 & 0 & 0 \\
0 & 1 & 0 & e^{\prime \prime} \\
0 & 0 & 1 & 0 \\
0 & 0 & 0 & 1
\end{array}\right)
$$

Since $\left[h_{1}, h_{2}\right]=h_{3}^{n_{1}} h_{4}^{n_{2}} C^{n_{3}}$ for some integers $n_{1}, n_{2}, n_{3}$, we obtain that $d^{\prime} f \quad \vdots \quad e^{\prime \prime}$ and $d^{\prime} a \vdots b^{\prime}$. Clearly we can generate $H$ with parameters of generators satisfying $|b|<\left|b^{\prime \prime}\right|,|e|<\left|e^{\prime \prime}\right|,\left|b^{\prime}\right|<\left|b^{\prime \prime}\right|,\left|e^{\prime}\right|<\left|e^{\prime \prime}\right|$.

We have

$$
\begin{gathered}
\chi\left(\left[h_{1}, h_{2}\right]\right)=w^{\frac{-d^{\prime} f}{e^{\prime \prime}}} z^{\frac{-d^{\prime} a}{b^{\prime \prime}}} \lambda^{-a d^{\prime} f-a d^{\prime} f^{\prime}+a e^{\prime}+b f^{\prime}-b^{\prime} f}=1, \\
\chi\left(\left[h_{1}, h_{3}\right]\right)=\lambda^{-f b^{\prime \prime}}=1, \quad \chi\left(\left[h_{1}, h_{4}\right]\right)=\lambda^{a e^{\prime \prime}}=1, \\
\chi\left(\left[h_{2}, h_{3}\right]\right)=\lambda^{-f^{\prime} b^{\prime \prime}}=1 .
\end{gathered}
$$

Since $d^{\prime} f \vdots e^{\prime \prime}, d^{\prime} a \vdots b^{\prime}$ and $\lambda^{\mathrm{GCD}\left(f, f^{\prime}\right) b^{\prime \prime}}=\lambda^{a e^{\prime \prime}}=1$, we have $\lambda^{-a d^{\prime} f-a d^{\prime} f^{\prime}}=1$. Then

$$
\chi\left(\left[h_{1}, h_{2}\right]\right)=w^{\frac{-d^{\prime} f}{e^{\prime \prime}}} z^{\frac{-d^{\prime} a}{b^{\prime \prime}}} \lambda^{-a d^{\prime} f-a d^{\prime} f^{\prime}+a e^{\prime}+b f^{\prime}-b^{\prime} f}=w^{\frac{-d^{\prime} f}{e^{\prime \prime}}} z^{\frac{-d^{\prime} a}{b^{\prime \prime}}} \lambda^{a e^{\prime}+b f^{\prime}-b^{\prime} f}=1 .
$$

The quotient $N_{G}(H) / H$ is generated by

$$
g=\left(\begin{array}{cccc}
1 & 0 & 0 & 0 \\
0 & 1 & 0 & 0 \\
0 & 0 & 1 & \tilde{f} \\
0 & 0 & 0 & 1
\end{array}\right)
$$

where $\tilde{f}$ is the minimal natural number such that $\tilde{f} d^{\prime}$ is divisible by $e^{\prime \prime}$.

The generator $g$ acts on a character $\chi$ as follows:

$$
\begin{gathered}
\chi^{g}\left(h_{1}\right)=\chi\left(h_{1}\right) \lambda^{-b \tilde{f}}, \quad \chi^{g}\left(h_{2}\right)=\chi\left(h_{2}\right) \chi\left(h_{4}\right)^{-\frac{d^{\prime} \tilde{f}}{e^{\prime \prime}}} \lambda^{-b^{\prime} \tilde{f}} \\
\text { and } \\
\chi^{g}\left(h_{3}\right)=\chi\left(h_{3}\right) \lambda^{-b^{\prime \prime} \tilde{f}}, \quad \chi^{g}\left(h_{4}\right)=\chi\left(h_{4}\right) .
\end{gathered}
$$

Then from irreducibility criterion $S(H, \chi)=H$ we obtain that the central character $\chi(C)$ is a root of unity of order $N$, where $N=$ $\operatorname{GCD}\left(f^{\prime} b^{\prime \prime}, a e^{\prime \prime}, f b^{\prime \prime}\right)=1$.

From the equation 8.3 , we obtain conditions for $w$ and $z$ defining $N_{2}$ and $N_{3}$. If both $z$ and $w$ are roots of unity, then the weight pair extends to the one with $\operatorname{rk}_{1}(H)=3$. Then for all three cases of $\phi\left(S_{1}\right), \phi\left(S_{2}\right)$ and $\phi\left(S_{3}\right)$ we have $Z_{2,2 ; H} \subseteq\left\{\mathbb{C}^{*} \times \mathbb{C}^{*} \times \mu_{\infty} \times\left\{\mathbb{C}^{*} \backslash \mu_{\infty}\right\} \times \mu_{\infty}\right\}$ (concrete formulas are in Lemma 7.1 formulation).

(ii). If $H \in \phi\left(S_{4}\right)$, then a subgroup $H$ may be generated as follows: 


$$
\begin{array}{rlrl}
h_{1} & =\left(\begin{array}{llll}
1 & a & b & 0 \\
0 & 1 & 0 & e \\
0 & 0 & 1 & 0 \\
0 & 0 & 0 & 1
\end{array}\right), & h_{2}=\left(\begin{array}{cccc}
1 & 0 & b^{\prime} & 0 \\
0 & 1 & 0 & e^{\prime} \\
0 & 0 & 1 & f^{\prime} \\
0 & 0 & 0 & 1
\end{array}\right), \\
h_{3}=\left(\begin{array}{llll}
1 & 0 & b^{\prime \prime} & 0 \\
0 & 1 & 0 & 0 \\
0 & 0 & 1 & 0 \\
0 & 0 & 0 & 1
\end{array}\right), & h_{4}=\left(\begin{array}{cccc}
1 & 0 & 0 & 0 \\
0 & 1 & 0 & e^{\prime \prime} \\
0 & 0 & 1 & 0 \\
0 & 0 & 0 & 1
\end{array}\right)
\end{array}
$$

Then we have

$$
\begin{gathered}
\chi\left(\left[h_{1}, h_{2}\right]\right)=\lambda^{a e^{\prime}+b f^{\prime}}=1, \\
\chi\left(\left[h_{1}, h_{4}\right]\right)=\lambda^{a e^{\prime \prime}}=1, \\
\chi\left(\left[h_{2}, h_{3}\right]\right)=\lambda^{-f^{\prime} b^{\prime \prime}}=1 .
\end{gathered}
$$

The quotient $N_{G}(H) / H$ is generated by

$$
g=\left(\begin{array}{cccc}
1 & 0 & 0 & 0 \\
0 & 1 & \tilde{d} & 0 \\
0 & 0 & 1 & 1 \\
0 & 0 & 0 & 1
\end{array}\right),
$$

where $\tilde{d}$ is the minimal natural number such that $\tilde{d} a$ is divisible by $b^{\prime \prime}$ and $\tilde{d} f^{\prime}$ is divisible by $e^{\prime \prime}$.

The generator $g$ acts on a character $\chi$ as follows:

$$
\begin{gathered}
\chi^{g}\left(h_{1}\right)=\chi\left(h_{1}\right) \chi\left(h_{3}\right)^{-\frac{a \tilde{d}}{b^{\prime}}}, \quad \chi^{g}\left(h_{2}\right)=\chi\left(h_{2}\right) \chi\left(h_{4}\right)^{-\frac{f^{\prime} \tilde{d}}{e^{\prime \prime}}} \\
\text { and } \\
\chi^{g}\left(h_{3}\right)=\chi\left(h_{3}\right), \quad \chi^{g}\left(h_{4}\right)=\chi\left(h_{4}\right) .
\end{gathered}
$$

Then from irreducibility criterion $S(H, \chi)=H$ we obtain that the central character $\chi(C)$ is a root of unity of order $N$, which was defined earlier.

If both $z$ and $w$ are roots of unity, then the weight pair extends to the one with $\mathrm{rk}_{1}(H)=3$. Thus, if $H \in \phi\left(S_{4}\right)$, then $Z_{2,2 ; H}$ is bijective to $\left\{\mathbb{C}^{*} \times \mathbb{C}^{*} \times\left\{\mathbb{C}^{*} \backslash \mu_{\infty}\right\} \times \mathbb{C}^{*} \times \mu_{N}\right\}=\left\{(t, s, z, w, \lambda) \mid z\right.$ or $\left.w \notin \mu_{\infty}, \lambda \in \mu_{N}\right\}$. 
Let us denote by $Z_{2,2 ; H}^{(2)}=\{\{(t, s, z, w, \lambda) \mid t, \quad s \quad \in$ $\left.\left.\left(\mathbb{C}^{*}\right)^{2}, \quad \mid w^{\frac{-d^{\prime} f}{e^{\prime \prime}}} z^{\frac{-d^{\prime} a}{b^{\prime \prime}}} \lambda^{a e^{\prime}+b f^{\prime}-b^{\prime} f}=1, \quad \lambda \in \mu_{N}\right\} / \sim\right\}$ and by $Z_{2,2 ; H}^{(1)}=$ $\left\{(z, w, \lambda) \mid w^{\frac{-d^{\prime} f}{e^{\prime \prime}}} z^{\frac{-d^{\prime} a}{b^{\prime \prime}}} \lambda^{a e^{\prime}+b f^{\prime}-b^{\prime} f}=1, \lambda \in \mu_{N}\right\}$, where $\sim$ is defined in 2.12,

Corollary 8.4. If $H \in \phi\left(S_{1}\right)$, then the fiber $Z_{2,2 ; H}$ of $Z_{2,2}$ over a subgroup $H$ has iterated structure of a bundle, namely:

$$
Z_{2,2 ; H}^{(2)} \rightarrow Z_{2,2 ; H}^{(1)}
$$

We describe fibers of this bundle consecutively in coordinates $(t, s, z, w, \lambda)$ :

$$
\mathbb{C}^{*}, E_{w \frac{d^{\prime} \tilde{f}}{e^{\prime \prime}}}, C_{z, w}, \mu_{N} \cup \mathbb{C}^{*}, P_{w \frac{d^{\prime} \tilde{f}}{e^{\prime \prime}}}, C_{z, w, s i n g}, \mu_{N}
$$

If $H \in \phi\left(S_{2}\right)$, then the fibers $Z_{2,2 ; H}^{(2)} \rightarrow Z_{2,2 ; H}^{(1)}$ over $H$ are canonically bijective to:

$\mathbb{C}^{*}, E_{w}^{\frac{d^{\prime} \tilde{f}}{e^{\prime \prime}}}, \mu_{N_{2}},\left\{\mathbb{C}^{*} \backslash S^{1}\right\}, \mu_{a e^{\prime \prime}} \cup \mathbb{C}^{*}, P_{w \frac{d^{\prime} \tilde{f}}{e^{\prime \prime}}}, \mu_{N_{2}},\left\{S^{1} \backslash \mu_{\infty}\right\}, \mu_{a e^{\prime \prime}} ;$

If $H \in \phi\left(S_{3}\right)$, then the fibers $Z_{2,2 ; H}^{(2)} \rightarrow Z_{2,2 ; H}^{(1)}$ over $H$ are canonically bijective to:

$E_{z \frac{d^{\prime} a}{b^{\prime \prime}}}, \mathbb{C}^{*},\left\{\mathbb{C}^{*} \backslash S^{1}\right\}, \mu_{N_{3}}, \mu_{f^{\prime} b^{\prime \prime}} \cup P_{z^{\frac{d^{\prime} a}{b^{\prime \prime}}}}, \mathbb{C}^{*},\left\{S^{1} \backslash \mu_{\infty}\right\}, \mu_{N_{3}}, \mu_{f^{\prime} b^{\prime \prime}}$

If $H \in \phi\left(S_{4}\right)$, then the fibers $Z_{2,2 ; H}^{(2)} \rightarrow Z_{2,2 ; H}^{(1)}$ over $H$ are canonically bijective to:

$$
\begin{aligned}
& E_{z^{\frac{d^{\prime} a}{b^{\prime \prime}}}}, E_{w \frac{d^{\prime} \tilde{f}}{e^{\prime \prime}}},\left\{\mathbb{C}^{*} \backslash S^{1}\right\},\left\{\mathbb{C}^{*} \backslash S^{1}\right\}, \mu_{N} \cup P_{z^{\frac{d^{\prime} a}{b^{\prime \prime}}}}, E_{w} \underbrace{}_{w^{\frac{d^{\prime} \tilde{f}}{e^{\prime \prime}}}},\left\{S^{1} \backslash \mu_{\infty}\right\},\left\{\mathbb{C}^{*} \backslash S^{1}\right\}, \mu_{N} \cup \\
& E_{z^{\frac{d^{\prime} a}{b^{\prime \prime}}}}, P_{w^{\frac{d^{\prime} \tilde{f}}{e^{\prime \prime}}}},\left\{\mathbb{C}^{*} \backslash S^{1}\right\},\left\{S^{1} \backslash \mu_{\infty}\right\}, \mu_{N} \cup P_{z^{\frac{d^{\prime} a}{b^{\prime \prime}}}}, P_{w}^{\frac{d^{\prime} \tilde{f}}{e^{\prime \prime}}},\left\{S^{1} \backslash \mu_{\infty}\right\},\left\{S^{1} \backslash \mu_{\infty}\right\}, \mu_{N} \cup \\
& \mathbb{C}^{*}, E_{w^{\frac{d^{\prime} \tilde{f}}{e^{\prime \prime}}}}, \mu_{\infty},\left\{\mathbb{C}^{*} \backslash S^{1}\right\}, \mu_{N} \cup E_{z^{\frac{d^{\prime} a}{b^{\prime \prime}}}}, \mathbb{C}^{*},\left\{\mathbb{C}^{*} \backslash S^{1}\right\}, \mu_{\infty}, \mu_{N} \cup \\
& \mathbb{C}^{*}, P_{w}{ }_{\frac{d^{\prime} \tilde{f}}{e^{\prime \prime}}}, \mu_{\infty},\left\{S^{1} \backslash \mu_{\infty}\right\}, \mu_{N} \cup P_{z^{\frac{d^{\prime} a}{b^{\prime \prime}}}}, \mathbb{C}^{*},\left\{S^{1} \backslash \mu_{\infty}\right\}, \mu_{\infty}, \mu_{N} .
\end{aligned}
$$

Proof. Follows directly from $N_{G}(H) / H$ action on a character $\chi$ of $H$ above.

Lemma 8.5. If $H \in \Sigma_{2,2}$, then there are only subgroups, which are $F$ equivalent to $H$ (finitely many subgroups).

Fibers $Z_{2,2 ; H_{1}}$ and $Z_{2,2 ; H_{2}}$ over equivalent subgroups $H_{1}$ and $H_{2}$ may be identified canonically. 
Proof. Since $N_{G}\left(H_{1}^{*}\right)=G$, by Proposition 2.16](ii)] we need to consider only equivalent irreducible weight pairs $\left(H_{2}, \chi_{2}\right)$ such that $H_{1}^{*}=H_{2}^{*}$ and $H_{1} \neq H_{2}$.

Values of a character $\chi_{1}$ in this case have to satisfy the following conditions:

$$
\begin{gathered}
\lambda^{f^{\prime} b^{\prime \prime}}=\lambda^{f b^{\prime \prime}}=\lambda^{a e^{\prime \prime}}=1, \\
w^{\frac{-d^{\prime} f}{e^{\prime \prime}}} z^{\frac{-d^{\prime} a}{b^{\prime \prime}}} \lambda^{a e^{\prime}+b f^{\prime}-b^{\prime} f}=1 .
\end{gathered}
$$

Let us denote $\operatorname{GCD}\left(f^{\prime} b^{\prime \prime}, f b^{\prime \prime}, a e^{\prime \prime}\right)$ by $N$.

We can only possibly extract roots from generators $h_{1}, h_{2}, h_{3}$, and $h_{4}$. If there is $g_{1} \in G \backslash H_{1}$ such that $g_{1}^{k_{1}}=h_{1}$, or $g_{2} \in G \backslash H_{1}$ such that $g_{2}^{k_{2}}=h_{2}$, then by Proposition [2.16(ii)] for any divisor $m_{1}$ of $k_{1}$ or $m_{2}$ of $k_{2}$, there exists a finite set of exponents of other generators such that $S\left(H_{2}, \chi_{2}\right)=H_{2}$. Similarly, for any divisor $m_{3}$ of $b^{\prime}$ and any divisor $m_{4}$ of $e^{\prime}$, there also exists a finite set of corresponding exponents. We omit concrete expressions of parameters of equivalent irreducible weight pairs due to their cumbersomeness.

Since $a, f, d^{\prime}, f^{\prime}, b^{\prime \prime}, e^{\prime \prime}$ are fixed, once we fixed the exponents of generators of $H_{1}$, there are only parameters $b, b^{\prime}<\left|b^{\prime \prime}\right|$ and $e, e^{\prime}<\left|e^{\prime \prime}\right|$ which can be varied to produce equivalent irreducible weight pairs. Since we have

$$
w^{\frac{-d^{\prime} f}{e^{\prime \prime}}} z^{\frac{-d^{\prime} a}{b^{\prime \prime}}} \lambda^{a e^{\prime}+b f^{\prime}-b^{\prime} f}=1,
$$

the expression $a e^{\prime}+b f^{\prime}-b^{\prime} f$ can not be changed modulo $N$, since $\lambda^{N}=1$. Thus, if we replace $b, b^{\prime}<\left|b^{\prime \prime}\right|$ and $e, e^{\prime}<\left|e^{\prime \prime}\right|$ by $\tilde{b}, \tilde{b^{\prime}}<\left|b^{\prime \prime}\right|$ and $\tilde{e}, \tilde{e^{\prime}}<\left|e^{\prime \prime}\right|$ such that

$$
\left(a e^{\prime}+b f^{\prime}-b^{\prime} f\right) \equiv\left(a \tilde{e^{\prime}}+\tilde{b} f^{\prime}-\tilde{b^{\prime}} f\right)(\bmod N),
$$

then there exists a corresponding character $\chi_{2}$ of a corresponding subgroup $H_{2}$ such that $\left.\chi_{1}\right|_{H_{1} \cap H_{2}}=\left.\chi_{2}\right|_{H_{1} \cap H_{2}}$. All such weight pairs are also $F$-equivalent to the weight pair $\left(H_{1}, \chi_{1}\right)$.

\section{The CASE OF $\operatorname{rk}_{1}(H)=3, \operatorname{rk}_{2}(H)=2$.}

Let $S=\left\{\left(a, b, e, d^{\prime}, b^{\prime}, e^{\prime}, f^{\prime \prime}, b^{\prime \prime}, e^{\prime \prime}, b^{\prime \prime \prime}, e^{\prime \prime \prime}\right) \in \mathbb{Z}^{11} \mid a \neq 0, d^{\prime} \neq 0, f^{\prime \prime} \neq\right.$ $0, \quad b^{\prime \prime \prime} \neq 0, \quad e^{\prime \prime \prime} \neq 0, \quad|b|<\left|b^{\prime \prime \prime}\right|,|e|<\left|e^{\prime \prime \prime}\right|,\left|b^{\prime}\right|<\left|b^{\prime \prime \prime}\right|,\left|e^{\prime}\right|<\left|e^{\prime \prime \prime}\right|,\left|b^{\prime \prime}\right|<$ $\left.\left|b^{\prime \prime \prime}\right|,\left|e^{\prime \prime}\right|<\left|e^{\prime \prime \prime}\right|\right\}$.

Let us denote $\operatorname{GCD}\left(f^{\prime \prime} b^{\prime \prime \prime}, a e^{\prime \prime}+b f^{\prime \prime}, a e^{\prime \prime \prime}\right)$ by $N_{3}$, and let $\lambda^{N_{3}}=1$. Let $N_{1}$ be a minimal natural number such that $z^{N_{1}}=1$, if $z^{\frac{-d^{\prime} a}{b^{\prime \prime \prime}}} \lambda^{-a e^{\prime}}=1$. Let $N_{2}$ be a minimal natural number such that $w^{N_{2}}=1$, if $w^{\frac{d^{\prime} f^{\prime \prime \prime}}{e^{\prime \prime \prime}}} \lambda^{b^{\prime} f^{\prime \prime}}=1$. 
Lemma 9.1. There is a canonical bijection $\phi$ from $S$ to $\Sigma_{3,2}$. It maps a tuple $\left(a, b, e, d^{\prime}, b^{\prime}, e^{\prime}, f^{\prime \prime}, b^{\prime \prime}, e^{\prime \prime}, b^{\prime \prime \prime}, e^{\prime \prime \prime}\right)$ to a subgroup $H$, generated by the following matrices

$$
\begin{gathered}
h_{1}=\left(\begin{array}{llll}
1 & a & b & 0 \\
0 & 1 & 0 & e \\
0 & 0 & 1 & 0 \\
0 & 0 & 0 & 1
\end{array}\right), \quad h_{2}=\left(\begin{array}{cccc}
1 & 0 & b^{\prime} & 0 \\
0 & 1 & d^{\prime} & e^{\prime} \\
0 & 0 & 1 & 0 \\
0 & 0 & 0 & 1
\end{array}\right), \quad h_{3}=\left(\begin{array}{cccc}
1 & 0 & b^{\prime \prime} & 0 \\
0 & 1 & 0 & e^{\prime \prime} \\
0 & 0 & 1 & f^{\prime \prime} \\
0 & 0 & 0 & 1
\end{array}\right), \\
h_{4}=\left(\begin{array}{cccc}
1 & 0 & b^{\prime \prime \prime} & 0 \\
0 & 1 & 0 & 0 \\
0 & 0 & 1 & 0 \\
0 & 0 & 0 & 1
\end{array}\right), \quad h_{5}=\left(\begin{array}{cccc}
1 & 0 & 0 & 0 \\
0 & 1 & 0 & e^{\prime \prime \prime} \\
0 & 0 & 1 & 0 \\
0 & 0 & 0 & 1
\end{array}\right), \quad C=\left(\begin{array}{cccc}
1 & 0 & 0 & 1 \\
0 & 1 & 0 & 0 \\
0 & 0 & 1 & 0 \\
0 & 0 & 0 & 1
\end{array}\right) .
\end{gathered}
$$

If $H \in \phi(S)$, we can extend the bijection $\phi$ to the map from

$\mathbb{C}^{*} \times \mathbb{C}^{*} \times \mathbb{C}^{*} \times \mu_{N_{1}} \times \mu_{N_{2}} \times \mu_{N_{3}}=\left\{(t, r, s, z, w, \lambda) \mid z \in \mu_{N_{1}}, w \in \mu_{N_{2}}, \lambda \in \mu_{N_{3}}\right\}$

to $Y_{3,2 ; H}$, which is defined as follows:

$$
t=\chi\left(h_{1}\right), \quad r=\chi\left(h_{2}\right), \quad s=\chi\left(h_{3}\right), \quad z=\chi\left(h_{4}\right), \quad w=\chi\left(h_{5}\right), \quad \lambda=\chi(C) .
$$

Proof. If $H \in \Sigma_{3,2}$, then $H$ may be generated as follows:

$$
\begin{gathered}
h_{1}=\left(\begin{array}{cccc}
1 & a & b & 0 \\
0 & 1 & 0 & e \\
0 & 0 & 1 & 0 \\
0 & 0 & 0 & 1
\end{array}\right), \quad h_{2}=\left(\begin{array}{cccc}
1 & 0 & b^{\prime} & 0 \\
0 & 1 & d & e^{\prime} \\
0 & 0 & 1 & 0 \\
0 & 0 & 0 & 1
\end{array}\right), \quad h_{3}=\left(\begin{array}{cccc}
1 & 0 & b^{\prime \prime} & 0 \\
0 & 1 & 0 & e^{\prime \prime} \\
0 & 0 & 1 & f \\
0 & 0 & 0 & 1
\end{array}\right), \\
h_{4}=\left(\begin{array}{cccc}
1 & 0 & b^{\prime \prime \prime} & 0 \\
0 & 1 & 0 & 0 \\
0 & 0 & 1 & 0 \\
0 & 0 & 0 & 1
\end{array}\right), \quad h_{5}=\left(\begin{array}{cccc}
1 & 0 & 0 & 0 \\
0 & 1 & 0 & e^{\prime \prime \prime} \\
0 & 0 & 1 & 0 \\
0 & 0 & 0 & 1
\end{array}\right) .
\end{gathered}
$$

Then we have

$$
\begin{aligned}
& \chi\left(\left[h_{1}, h_{2}\right]\right)=z^{\frac{-d^{\prime} a}{b^{\prime \prime \prime}}} \lambda^{-a e^{\prime}}=\chi\left(h_{4}\right)^{N_{1}}=1, \\
& \chi\left(\left[h_{2}, h_{3}\right]\right)=w^{\frac{d^{\prime} f^{\prime \prime}}{e^{\prime \prime \prime}}} \lambda^{b^{\prime} f^{\prime \prime}}=\chi\left(h_{5}\right)^{N_{2}}=1 .
\end{aligned}
$$

We also have $\chi(C)^{N_{3}}=\lambda^{N_{3}}=1$, because

$$
\begin{gathered}
\chi\left(\left[h_{1}, h_{3}\right]\right)=\lambda^{-a e^{\prime \prime}-b f^{\prime \prime}}=1, \\
\chi\left(\left[h_{3}, h_{4}\right]\right)=\lambda^{b^{\prime \prime \prime} f^{\prime \prime}}=1
\end{gathered}
$$




$$
\chi\left(\left[h_{1}, h_{5}\right]\right)=\lambda^{-a e^{\prime \prime \prime}}=1 .
$$

In this case, the quotient $N_{G}(H) / H$ is clearly finite, and since $N_{1}, N_{2}$ and $N_{3}$ are chosen to be minimal natural numbers such that $z^{N_{1}}=w^{N_{2}}=\lambda^{N_{3}}=1$, the action of $N_{G}(H) / H$ on characters is free. Hence, $S(H, \chi)=H$, and the corresponding representations are irreducible and finite-dimensional. Let us note that this case is the only one of finite-dimensional irreducible representations, all the others (cases $1-5$ ) refer to the infinite-dimensional ones.

Corollary 9.2. If $H \in \Sigma_{3,2}$, then the fiber $Z_{3,2 ; H}$ over $H$ is canonically bijective to:

$$
\mathbb{C}^{*} \times \mathbb{C}^{*} \times \mathbb{C}^{*} \times \mu_{N_{1}} \times \mu_{N_{2}} \times \mu_{N_{3}}
$$

Proof. Since in this case $N_{G}(H) / H$ is finite, its action on a character $\chi$ does not change a conformal class of $Y_{3,2 ; H}$.

Lemma 9.3. If $H \in \Sigma_{3,2}$, then there are only subgroups, which are $F$ equivalent to $H$ (finitely many subgroups).

Fibers $Z_{3,2 ; H_{1}}$ and $Z_{3,2 ; H_{2}}$ over equivalent subgroups $H_{1}$ and $H_{2}$ may be identified canonically.

Proof. If $H_{1} \in \Sigma_{3,2}$, then $H_{1}^{*}=G$. By Proposition [2.16](ii)] we need to consider only equivalent irreducible weight pairs $\left(H_{2}, \chi_{2}\right)$ such that $H_{1}^{*}=H_{2}^{*}$ and $H_{1} \neq H_{2}$.

Conditions for characters in this case are the following ones:

$$
\begin{gathered}
\lambda^{f^{\prime \prime} b^{\prime \prime \prime}}=\lambda^{a e^{\prime \prime}+b f^{\prime \prime}}=\lambda^{a e^{\prime \prime \prime}}=1, \\
w^{\frac{d^{\prime} f^{\prime \prime}}{e^{\prime \prime \prime}}} \lambda^{b^{\prime} f^{\prime \prime}}=1 \quad \text { and } \quad z^{\frac{-d^{\prime} a}{b^{\prime \prime \prime}}} \lambda^{-a e^{\prime}}=1 .
\end{gathered}
$$

Let us denote $\operatorname{GCD}\left(f^{\prime \prime} b^{\prime \prime \prime}, a e^{\prime \prime}+b f^{\prime \prime}, a e^{\prime \prime \prime}\right)$ by $N_{3}$.

We can possibly extract roots from generators $h_{1}, h_{2}, h_{3}, h_{4}$, and $h_{5}$. If there is $g_{1} \in G \backslash H_{1}$ such that $g_{1}^{k_{1}}=h_{1}$, or $g_{2} \in G \backslash H_{1}$ such that $g_{2}^{k_{2}}=h_{2}$, or $g_{3} \in G \backslash H_{1}$ such that $g_{3}^{k_{3}}=h_{3}$, then by Proposition [2.16[(ii)] for any divisor $m_{1}$ of $k_{1}$ or $m_{2}$ of $k_{2}$ or $m_{3}$ of $k_{3}$, there exists a finite set of exponents of other generators such that $S\left(H_{2}, \chi_{2}\right)=H_{2}$. Similarly, for any divisor $m_{4}$ of $b^{\prime \prime \prime}$ and any divisor $m_{5}$ of $e^{\prime \prime \prime}$, there also exists a finite set of corresponding exponents.

Since $a, d^{\prime}, f^{\prime \prime}, b^{\prime \prime \prime}, e^{\prime \prime \prime}$ are fixed, once we fixed the exponents of generators of $H_{1}$, there are only parameters $b, b^{\prime}, b^{\prime \prime}<\left|b^{\prime \prime \prime}\right|$ and $e, e^{\prime}, e^{\prime \prime}<\left|e^{\prime \prime \prime}\right|$ which can be varied to produce equivalent irreducible weight pairs. Since we have

$$
w^{\frac{d^{\prime} f^{\prime \prime}}{e^{\prime \prime \prime}}} \lambda^{b^{\prime} f^{\prime \prime}}=1 \quad \text { and } \quad z^{\frac{-d^{\prime} a}{b^{\prime \prime \prime}}} \lambda^{-a e^{\prime}}=1 \quad \text { and } \quad \lambda^{a e^{\prime \prime}+b f^{\prime \prime}}=1,
$$


the expressions $b^{\prime} f^{\prime \prime},-a e^{\prime}$ and $a e^{\prime \prime}+b f^{\prime \prime}$ can not be changed modulo $N_{3}$, since $\lambda^{N_{3}}=1$. So if we replace $b, b^{\prime}, b^{\prime \prime}<\left|b^{\prime \prime \prime}\right|$ and $e, e^{\prime}, e^{\prime \prime}<\left|e^{\prime \prime \prime}\right|$ by $\tilde{b}, \tilde{b^{\prime}}, \tilde{b^{\prime \prime}}<\left|b^{\prime \prime \prime}\right|$ and $\tilde{e}, \tilde{e^{\prime}}, \tilde{e^{\prime \prime}}<\left|e^{\prime \prime \prime}\right|$ such that expressions above are unchanged modulo $N_{3}$, then there exists a corresponding character $\chi_{2}$ of a corresponding subgroup $H_{2}$ such that $\left.\chi_{1}\right|_{H_{1} \cap H_{2}}=\left.\chi_{2}\right|_{H_{1} \cap H_{2}}$. All such weight pairs are also $F$-equivalent to the weight pair $\left(H_{1}, \chi_{1}\right)$.

\section{The MAIN RESUlT}

Thus, we have finally obtained:

Theorem 10.1. There is a one-to-one correspondence between the following spaces:

1. The union of the total spaces of the following bundles: $X_{1,1} \rightarrow \Xi_{1,1}$, $X_{2,0} \rightarrow \Xi_{2,0}, X_{2,1} \rightarrow \Xi_{2,1}, X_{1,2} \rightarrow \Xi_{1,2}, X_{2,2} \rightarrow \Xi_{2,2}$, and $X_{3,2} \rightarrow \Xi_{3,2}$.

2. A coarse moduli space of irreducible representations for the group of unipotent matrices of order 4 with integer entries which have finite weight.

A map from $X_{r_{1}, r_{2}} \rightarrow \Xi_{r_{1}, r_{2}}$ to the set of irreducible monomial representations is defined as follows:

$$
(H, \chi) \longmapsto \operatorname{ind}_{H}^{G}(\chi) .
$$

The fibers of these bundles are given in Corollaries 4.5, 5.3, 6.3, 7.2, 8.4, 9.2 and Lemmas 4.6, 5.4, 6.4, 7.3, 8.5, 9.3. The definition of the bundle $X_{r_{1}, r_{2}} \rightarrow$ $\Xi_{r_{1}, r_{2}}$ is given in 2.14.

The fibers of the bundle $Z_{\mathrm{rk}_{1}, \mathrm{rk}_{2}} \rightarrow \Sigma_{\mathrm{rk}_{1}, \mathrm{rk}_{2}}$ are given in Table 1 below (see the corresponding definition in 2.12). 
Acknoledgements. The author is grateful to S. Gorchinskiy for useful discussions and suggestions. The author was supported by the National Centre of Competence in Research "SwissMAP The Mathematics of Physics" of the Swiss National Science Foundation. The author is also grateful for hospitality and excellent working conditions to the Max Planck Institute for Mathematics, where a part of the work was done.

\section{REFERENCES}

[1] S. A. Arnal, A. N. Parshin, On irreducible representations of discrete Heisenberg groups, Mat. Zametki, 92:3 (2012), 323-330; translation in Math. Notes 92:3 (2012), 295-301.

[2] I. V. Beloshapka, S. O. Gorchinskiy, Irreducible representations of finitely generated nilpotent groups, Mat. Sb. 207:1 (2016), 45-72.

[3] I. V. Beloshapka, On representations of a Heisenberg group of matrices with one integer and two real coefficients, Math. Surveys, 69:5 (2014), 921-923.

[4] I. V. Beloshapka, On irreducible representations with finite weight of a certain discrete nilpotent group, Math. Surveys, 70:4 (2015), 777-778.

[5] J. N. Bernstein, A. V. Zelevinskii, Representations of the group $\mathrm{GL}(n, F)$, where $F$ is a local non-Archimedean field, Uspehi Mat. Nauk, 31:3 (189), (1976), 5-70; translation in Russian Math. Surveys, 31:3 (1976), 1-68.

[6] I. D. Brown, Representation of finitely generated nilpotent groups, Pacific J. Math., 45:1 (1973), 13-26.

[7] D. Boyarchenko, M. Sabitova, The orbit method for profinite groups and p-adic analogue of Brown's theorem, e-print arXiv: mathRT/0608126.

[8] A. A. Kirillov, Lectures on the orbit method, Graduate Studies in Mathematics, 64 Providence, RI: Amer. Math.Soc. (2004), 408 p.

[9] A.N. Parshin, On holomorphic representations of discrete Heisenberg groups, Funktsional. Anal. i Prilozhen., 44:2 (2010), 92-96; translation in Funct. Anal. Appl., 44:2 (2010), 156-159.

[10] A. N.Parshin, Representations of higher adelic groups and arithmetic, Proceedings of the International Congress of Mathematicians, I (2010), 362-392, Hindustan Book Agency, New Delhi.

ETH ZuRICH, Switzerland

E-mail address: iuliya.beloshapka@math.ethz.ch 
TABLE 1. Fibers of the bundle $Z_{\mathrm{rk}_{1}, \mathrm{rk}_{2}} \rightarrow \Sigma_{\mathrm{rk}_{1}, \mathrm{rk}_{2}}$

\begin{tabular}{|c|c|c|c|c|c|c|c|c|}
\hline $\mathrm{rk}_{1}$ & $\mathrm{rk}_{2}$ & Subset & $t$ & $r$ & $s$ & $z$ & $w$ & $\bar{\lambda}$ \\
\hline 1 & 1 & $\phi\left(S_{1}\right)$ & $T_{z^{d} \lambda^{a^{\prime} e+f^{\prime} b+a^{\prime} f^{\prime} d}, a}$ & & & $E_{\lambda^{2 a^{\prime} f^{\prime}}} \backslash \mu_{\infty}$ & & $\mathbb{C}^{*} \backslash S^{1}$ \\
\hline 1 & 1 & $\phi\left(S_{1}\right)$ & $T_{z^{d} \lambda^{a^{\prime} e+f^{\prime} b+a^{\prime} f^{\prime} d}, a}$ & & & $P_{\lambda^{2 a^{\prime} f^{\prime}}} \backslash \mu_{\infty}$ & & $S^{1} \backslash \mu_{\infty}$ \\
\hline 1 & 1 & $\phi\left(S_{1}\right)$ & $E_{\lambda} \operatorname{GCD}\left(a^{\prime} e+f^{\prime} b+a^{\prime} f^{\prime} d, a\right)$ & & & $\mu_{\infty}$ & & $\mathbb{C}^{*} \backslash S^{1}$ \\
\hline 1 & 1 & $\phi\left(S_{1}\right)$ & $P_{\lambda \mathrm{GCD}\left(a^{\prime} e+f^{\prime} b+a^{\prime} f^{\prime} d, a\right)}$ & & & $\mu_{\infty}$ & & $S^{1} \backslash \mu_{\infty}$ \\
\hline 1 & 1 & $\phi\left(S_{1}\right)$ & 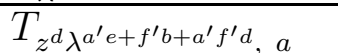 & & & $E_{\lambda^{2 a^{\prime} f^{\prime}}} \backslash \mu_{\infty}$ & & $\mathbb{C}^{*} \backslash S^{1}$ \\
\hline 1 & 1 & $\phi\left(S_{1}\right)$ & 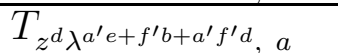 & & & $P_{\lambda^{2 a^{\prime} f^{\prime}}} \backslash \mu_{\infty}$ & & $S^{1} \backslash \mu_{\infty}$ \\
\hline 1 & 1 & $\phi\left(S_{1}\right)$ & $E_{\lambda \mathrm{GCD}\left(a^{\prime} e+f^{\prime} b+a^{\prime} f^{\prime} d, a\right)}$ & & & $\mu_{\infty}$ & & $\mathbb{C}^{*} \backslash S^{1}$ \\
\hline 1 & 1 & $\phi\left(S_{1}\right)$ & $P_{\lambda} \operatorname{GCD}\left(a^{\prime} e+f^{\prime} b+a^{\prime} f^{\prime} d, a\right)$ & & & $\mu_{\infty}$ & & $S^{1} \backslash \mu_{\infty}$ \\
\hline 1 & 1 & $\phi\left(S_{2}\right)$ & 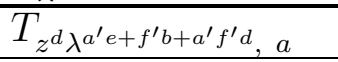 & & & $E_{\lambda^{2 a^{\prime} f^{\prime}}} \backslash \mu_{\infty}$ & & $\mathbb{C}^{*} \backslash S^{1}$ \\
\hline 1 & 1 & $\phi\left(S_{2}\right)$ & 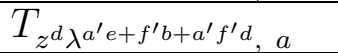 & & & $P_{\lambda^{2 a^{\prime} f^{\prime}}} \backslash \mu_{\infty}$ & & $S^{1} \backslash \mu_{\infty}$ \\
\hline 1 & 1 & $\phi\left(S_{2}\right)$ & $E_{\lambda} \operatorname{GCD}\left(a^{\prime} e+f^{\prime} b+a^{\prime} f^{\prime} f_{d, a}\right.$ & & & $\mu_{\infty}$ & & $\mathbb{C}^{*} \backslash S^{1}$ \\
\hline 1 & 1 & $\phi\left(S_{2}\right)$ & $P_{\lambda \mathrm{GCD}\left(a^{\prime} e+f^{\prime} b+a^{\prime} f^{\prime} d, a\right)}$ & & & $\mu_{\infty}$ & & $S^{1} \backslash \mu_{\infty}$ \\
\hline 1 & 1 & $\phi\left(S_{2}\right)$ & 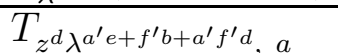 & & & $E_{\lambda^{2 a^{\prime} f^{\prime}}} \backslash \mu_{\infty}$ & & $\mathbb{C}^{*} \backslash S^{1}$ \\
\hline 1 & 1 & $\phi\left(S_{2}\right)$ & 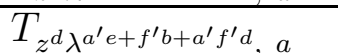 & & & $P_{\lambda_{\lambda^{2} f^{\prime} f^{\prime}}} \backslash \mu_{\infty}$ & & $S^{1} \backslash \mu_{\infty}$ \\
\hline 1 & 1 & $\phi\left(S_{2}\right)$ & $E_{\lambda \mathrm{GCD}\left(a^{\prime} e+f^{\prime} b+a^{\prime} f^{\prime} d, a\right)}$ & & & $\mu_{\infty}$ & & $\mathbb{C}^{*} \backslash S^{1}$ \\
\hline 1 & 1 & $\phi\left(S_{2}\right)$ & $P_{\lambda \mathrm{GCD}\left(a^{\prime} e+f^{\prime} b+a^{\prime} f^{\prime} d, a\right)}$ & & & $\mu_{\infty}$ & & $S^{1} \backslash \mu_{\infty}$ \\
\hline 1 & 1 & $\phi\left(S_{3}\right)$ & $\bar{T} \frac{z^{d} \lambda^{a^{\prime} e}, \lambda^{a}}{}$ & & & $\mathbb{C}^{*} \backslash S^{1}$ & & $\mathbb{C}^{*} \backslash S^{1}$ \\
\hline 1 & 1 & $\phi\left(S_{3}\right)$ & 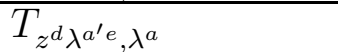 & & & $S^{1} \backslash \mu_{\infty}$ & & $\mathbb{C}^{*} \backslash S^{1}$ \\
\hline 1 & 1 & $\phi\left(S_{3}\right)$ & $T_{z^{d} \lambda^{a^{\prime} e}, \lambda^{a}}$ & & & $\mathbb{C}^{*} \backslash \mu_{\infty}$ & & $S^{1} \backslash \mu_{\infty}$ \\
\hline 1 & 1 & $\phi\left(S_{3}\right)$ & $E_{\lambda^{\operatorname{GCD}\left(a^{\prime} e, a\right)}}$ & & & $\mu_{\infty}$ & & $\mathbb{C}^{*} \backslash S^{1}$ \\
\hline 1 & 1 & $\phi\left(S_{3}\right)$ & $P_{\lambda \mathrm{GCD}\left(a^{\prime} e, a\right)}$ & & & $\mu_{\infty}$ & & $S^{1} \backslash \mu_{\infty}$ \\
\hline 1 & 1 & $\phi\left(S_{4}\right)$ & $E_{\lambda \mathrm{GCD}\left(a^{\prime} e+f^{\prime} b, a\right)}$ & & & $E_{\lambda^{2 a^{\prime} f^{\prime}}}$ & & $\mathbb{C}^{*} \backslash S^{1}$ \\
\hline 1 & 1 & $\phi\left(S_{4}\right)$ & $P_{\lambda \mathrm{GCD}\left(a^{\prime} e+f^{\prime} b, a\right)}$ & & & $P P_{\lambda^{2 a^{\prime} f^{\prime}}}$ & & $S^{1} \backslash \mu_{\infty}$ \\
\hline 1 & 1 & $\phi\left(N_{1}\right)$ & $\overline{T_{z^{a}, \lambda^{a}}}$ & & & $E_{\lambda} \backslash \mu_{\infty}$ & & $\mathbb{C}^{*} \backslash S^{1}$ \\
\hline 1 & 1 & $\phi\left(N_{1}\right)$ & $T_{z^{a}, \lambda^{a}}$ & & & $P_{\lambda} \backslash \mu_{\infty}$ & & $S^{1} \backslash \mu_{\infty}$ \\
\hline 1 & 1 & $\phi\left(N_{1}\right)$ & $E_{\lambda}$ & & & $\mu_{\infty}$ & & $\mathbb{C}^{*} \backslash S^{1}$ \\
\hline 1 & 1 & $\phi\left(N_{1}\right)$ & $P_{\lambda}$ & & & $\mu_{\infty}$ & & $S^{1} \backslash \mu_{\infty}$ \\
\hline 1 & 1 & $\phi\left(N_{2}\right)$ & $\overline{T_{z^{f}, \lambda^{f}}}$ & & & $E_{\lambda} \backslash \mu_{\infty}$ & & $\mathbb{C}^{*} \backslash S^{1}$ \\
\hline 1 & 1 & $\phi\left(N_{2}\right)$ & $T_{z^{f}, \lambda^{f}}$ & & & $P_{\lambda} \backslash \mu_{\infty}$ & & $S^{1} \backslash \mu_{\infty}$ \\
\hline 1 & 1 & $\phi\left(N_{2}\right)$ & $\overline{E_{\lambda}}$ & & & $\mu_{\infty}$ & & $\mathbb{C}^{*} \backslash S^{1}$ \\
\hline 1 & 1 & $\phi\left(N_{2}\right)$ & $\overline{P_{\lambda}}$ & & & $\mu_{\infty}$ & & $S^{1} \backslash \mu_{\infty}$ \\
\hline 2 & 0 & $\Sigma_{2,0}$ & $E_{\lambda f^{\prime}}$ & & $E_{\lambda^{a}}$ & & & $\mathbb{C}^{*} \backslash S^{1}$ \\
\hline 2 & 0 & $\Sigma_{2,0}$ & $P_{\lambda f^{\prime}}$ & & $P_{\lambda^{a}}$ & & & $S^{1} \backslash \mu_{\infty}$ \\
\hline 2 & 1 & $\Sigma_{2,1}$ & $E_{\lambda^{a}}$ & $\mathbb{C}^{*}$ & & $\mu_{a d^{\prime}}$ & & $\mathbb{C}^{*} \backslash S^{1}$ \\
\hline 2 & 1 & $\Sigma_{2,1}$ & $P_{\lambda^{a}}$ & $\mathbb{C}^{*}$ & & $\mu_{a d^{\prime}}$ & & $S^{1} \backslash \mu_{\infty}$ \\
\hline 1 & 2 & $\Sigma_{1,2} \backslash \phi(A)$ & $T_{z} \frac{a d^{\prime}}{b^{\prime}} w^{\frac{f d^{\prime}}{e^{\prime}}}, w^{\frac{f^{\prime} d}{e^{\prime}}} \lambda^{b f^{\prime}}$ & & & $\mathbb{C}^{*} \backslash \mu_{\infty}$ & $\mathbb{C}^{*} \backslash \mu_{\infty}$ & $\mu_{N}$ \\
\hline
\end{tabular}




\begin{tabular}{|c|c|c|c|c|c|c|c|c|}
\hline 1 & 2 & $\phi(A)$ & $T_{z, w}$ & & & $E_{\lambda} \backslash \mu_{\infty}$ & $E_{\lambda} \backslash \mu_{\infty}$ & $\mathbb{C}^{*} \backslash S^{1}$ \\
\hline 1 & 2 & $\phi(A)$ & $T_{z, w}$ & & & $P_{\lambda} \backslash \mu_{\infty}$ & $P_{\lambda} \backslash \mu_{\infty}$ & $P_{\lambda} \backslash \mu_{\infty}$ \\
\hline 1 & 2 & $\phi(A)$ & $E_{z}$ & & & $E_{\lambda} \backslash \mu_{\infty}$ & $E_{\lambda} \backslash \mu_{\infty}$ & $\mathbb{C}^{*} \backslash S^{1}$ \\
\hline 1 & 2 & $\phi(A)$ & $E_{z}$ & & & $P_{\lambda} \backslash \mu_{\infty}$ & $P_{\lambda} \backslash \mu_{\infty}$ & $S^{1} \backslash \mu_{\infty}$ \\
\hline 1 & 2 & $\phi(A)$ & $E_{w}$ & & & $E_{\lambda} \cap \mu_{\infty}$ & $E_{\lambda} \cap \mu_{\infty}$ & $\mathbb{C}^{*} \backslash S^{1}$ \\
\hline 1 & 2 & $\phi(A)$ & $E_{w}$ & & & $P_{\lambda} \cap \mu_{\infty}$ & $P_{\lambda} \cap \mu_{\infty}$ & $S^{1} \backslash \mu_{\infty}$ \\
\hline 1 & 2 & $\phi(A)$ & $\mathbb{C}^{*}$ & & & $E_{\lambda} \cap \mu_{\infty}$ & $E_{\lambda} \cap \mu_{\infty}$ & $\mathbb{C}^{*} \backslash S^{1}$ \\
\hline 1 & 2 & $\phi(A)$ & $\mathbb{C}^{*}$ & & & $P_{\lambda} \cap \mu_{\infty}$ & $P_{\lambda} \cap \mu_{\infty}$ & $S^{1} \backslash \mu_{\infty}$ \\
\hline 1 & 2 & $\phi(A)$ & $T_{z, w}$ & & & $\mathbb{C}^{*} \backslash \mu_{\infty}$ & $\mathbb{C}^{*} \backslash \mu_{\infty}$ & $\mu_{\infty}$ \\
\hline 1 & 2 & $\phi(A)$ & $T_{z, w}$ & & & $\mathbb{C}^{*} \backslash \mu_{\infty}$ & $\mathbb{C}^{*} \backslash \mu_{\infty}$ & $\mu_{\infty}$ \\
\hline 2 & 2 & $\phi\left(S_{1}\right)$ & $\mathbb{C}^{*}$ & & $E_{w} \frac{d^{\prime} \tilde{f}}{e^{\prime \prime}}$ & $C_{z, w}$ & $C_{z, w}$ & $\mu_{N}$ \\
\hline 2 & 2 & $\phi\left(S_{1}\right)$ & $\mathbb{C}^{*}$ & & $P_{\frac{d^{\prime} \tilde{f}}{e^{\prime \prime}}}$ & $C_{z, w, \operatorname{sing}}$ & $C_{z, w, s i n g}$ & $\mu_{N}$ \\
\hline 2 & 2 & $\phi\left(S_{1}\right)$ & $\mathbb{C}^{*}$ & & $E \frac{d^{\prime} \tilde{f}}{d \prime}$ & $C_{z, w}$ & $C_{z, w}$ & $\mu_{N}$ \\
\hline 2 & 2 & $\phi\left(S_{1}\right)$ & $\mathbb{C}^{*}$ & & $P_{\frac{d^{\prime} \tilde{f}}{d^{\prime \prime}}}$ & $C_{z, w, \operatorname{sing}}$ & $C_{z, w, \sin g}$ & $\mu_{N}$ \\
\hline 2 & 2 & $\phi\left(S_{2}\right)$ & $\mathbb{C}^{*}$ & & $E \frac{d^{\prime} \tilde{f}}{n}$ & $\mu_{N_{2}}$ & $\mathbb{C}^{*} \backslash S^{1}$ & $\mu_{a e^{\prime \prime}}$ \\
\hline 2 & 2 & $\phi\left(S_{2}\right)$ & $\mathbb{C}^{*}$ & & $P_{\frac{d^{\prime} \tilde{f}}{e^{\prime \prime}}}$ & $\mu_{N_{2}}$ & $S^{1} \backslash \mu_{\infty}$ & $\mu_{a e^{\prime \prime}}$ \\
\hline 2 & 2 & $\phi\left(S_{3}\right)$ & $E_{z \frac{d^{\prime} a}{b^{\prime \prime}}}$ & & $\mathbb{C}^{*}$ & $\mathbb{C}^{*} \backslash S^{1}$ & $\mu_{N_{3}}$ & $\mu_{f^{\prime} b^{\prime \prime}}$ \\
\hline 2 & 2 & $\phi\left(S_{3}\right)$ & $P_{\frac{d^{\prime} a}{b^{\prime \prime}}}$ & & $\mathbb{C}^{*}$ & $S^{1} \backslash \mu_{\infty}$ & $\mu_{N_{3}}$ & $\mu_{f^{\prime} b^{\prime \prime}}$ \\
\hline 2 & 2 & $\phi\left(S_{4}\right)$ & $E_{\frac{d^{\prime} a}{b^{\prime \prime}}}$ & & $E_{\frac{d^{\prime} \tilde{f}}{f^{\prime \prime}}}$ & $\mathbb{C}^{*} \backslash S^{1}$ & $\mathbb{C}^{*} \backslash S^{1}$ & $\mu_{N}$ \\
\hline 2 & 2 & $\phi\left(S_{4}\right)$ & $P_{z \frac{d^{\prime} a}{b^{\prime \prime}}}$ & & $E_{\frac{d^{\prime} \tilde{f}}{e^{\prime \prime}}}$ & $S^{1} \backslash \mu_{\infty}$ & $\mathbb{C}^{*} \backslash S^{1}$ & $\mu_{N}$ \\
\hline 2 & 2 & $\phi\left(S_{4}\right)$ & $E_{z \frac{d^{\prime} a}{b^{\prime \prime}}}$ & & $E_{\frac{d^{\prime} \tilde{f}}{e^{\prime \prime}}}$ & $\mathbb{C}^{*} \backslash S^{1}$ & $\mathbb{C}^{*} \backslash S^{1}$ & $\mu_{N}$ \\
\hline 2 & 2 & $\phi\left(S_{4}\right)$ & $P_{z} \frac{d^{\prime} a}{b^{\prime \prime}}$ & & $P_{w \frac{d^{\prime} \tilde{f}}{e^{\prime \prime}}}$ & $S^{1} \backslash \mu_{\infty}$ & $S^{1} \backslash \mu_{\infty}$ & $\overline{\mu_{N}}$ \\
\hline 2 & 2 & $\phi\left(S_{4}\right)$ & $\mathbb{C}^{*}$ & & $E_{w} \frac{d^{\prime} \tilde{f}}{e^{\prime \prime}}$ & $\mu_{\infty}$ & $\mathbb{C}^{*} \backslash S^{1}$ & $\mu_{N}$ \\
\hline 2 & 2 & $\phi\left(S_{4}\right)$ & $E_{\frac{d^{\prime} a}{b^{\prime \prime}}}$ & & $\mathbb{C}^{*}$ & $\mathbb{C}^{*} \backslash S^{1}$ & $\mu_{\infty}$ & $\mu_{N}$ \\
\hline 2 & 2 & $\phi\left(S_{4}\right)$ & $\mathbb{C}^{*}$ & & $P_{\frac{d^{\prime} \tilde{f}}{e^{\prime \prime}}}$ & $\mu_{\infty}$ & $S^{1} \backslash \mu_{\infty}$ & $\mu_{N}$ \\
\hline 2 & 2 & $\phi\left(S_{4}\right)$ & $P_{\frac{d^{\prime} a}{b^{\prime \prime}}}$ & & $\mathbb{C}^{*}$ & $S^{1} \backslash \mu_{\infty}$ & $\mu_{\infty}$ & $\mu_{N}$ \\
\hline 3 & 2 & $\Sigma_{3,2}$ & $\mathbb{C}^{*}$ & $\mathbb{C}^{*}$ & $\mathbb{C}^{*}$ & $\mu_{N_{1}}$ & $\mu_{N_{2}}$ & $\mu_{N_{3}}$ \\
\hline
\end{tabular}

